\title{
TORAL ACTIONS ON 4-MANIFOLDS AND THEIR CLASSIFICATIONS
}

\author{
M. HO KIM
}

\begin{abstract}
The existence of a cross-section is proved for some nonorientable 4-manifolds with a $T^{2}$-action. Two 4-manifolds with a $T^{2}$-action, which have the same previously known invariants, are constructed. By using a new homotopy invariant, they are proved to be homotopy inequivalent. Finally a stable diffeomorphism theorem is proved.
\end{abstract}

\section{INTRODUCTION}

P. Orlik and F. Raymond showed, in [OR, I], the following:

Suppose that $M$ is a 4-dimensional closed simply-connected manifold with an effective $T^{2}$-action. Then $M$ is an equivariant connected sum of $\mathbb{C P}^{2}, \overline{\mathbb{C P}}^{2}, S^{2} \times$ $S^{2}$ and $S^{4}$

In [OR, II], they studied some non-simply-connected manifolds with an effective $T^{2}$-action and proved that, if the manifolds have neither fixed points nor circle subgroups as stabilizers, then, in "almost all" cases, two manifolds are diffeomorphic if and only if they are equivariantly diffeomorphic up to an automorphism of $T^{2}$.

With the presence of fixed points or circle orbits, the techniques of a topological classification are quite different. Orlik and Raymond obtained an equivariant classification when $M$ has a fixed point, but there were three families of $T^{2}$-manifolds, called basic blocks. To obtain a topological classification of closed orientable $T^{2}$-manifolds with a fixed point, it was necessary to study these families of $T^{2}$-manifolds, which are described in terms of orbit spaces. For example, each of the manifolds of one family has the orbit space pictured in Figure 1 (see $\S 2$ for a description of this space and [OR, II]). They showed that

$$
\begin{array}{rlrl}
M \# k\left(S^{2} \times S^{2}\right) & =\left(S^{1} \times S^{3}\right) \#\left(S^{2} \times S^{2}\right) \# k\left(S^{2} \times S^{2}\right), & & \text { if } m n \text { is even, } \\
& =\left(S^{1} \times S^{3}\right) \# \mathbb{C P}^{2} \# \overline{\mathbb{C P}}^{2} \# k\left(S^{2} \times S^{2}\right), & \text { if } m n \text { is odd, }
\end{array}
$$

where $k$ is an integer. Whether or not the addition of the $k$ copies of $S^{2} \times S^{2}$ could be dropped was left unsettled.

Received by the editors July 22, 1989 and, in revised form, April 20, 1990.

1980 Mathematics Subject Classification (1985 Revision). Primary 57M99.

Key words and phrases. Toral action, orbit space, nonorientable, cross-section, fixed points, equivariant, stabilizers.

This paper represents a part of the author's doctoral dissertation written under the generous supervision of Professor Frank Raymond at the University of Michigan at Ann Arbor. The author is grateful to Professor Raymond for patient support and indebted to Professors J. Kister, S. Kojima and P. Scott for invaluable discussion. 


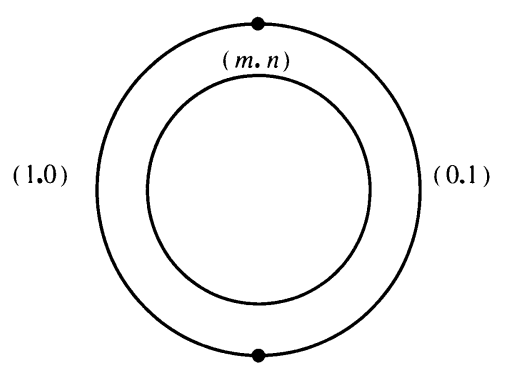

FIGURE 1. 2-dimensional annulus

P. Pao attacked these unsettled problems with elementary, but clever techniques and eliminated the necessity of stabilization of the above as well as the other two families in [P]. Furthermore, he proved the following theorem:

Suppose $M$ is a 4-dimensional orientable closed manifold with an effective $T^{2}$-action. If $M^{*}$ has a fixed point, then $M$ can be decomposed into a connected sum of copies of $S^{4}, S^{2} \times S^{2}, \mathbb{C P}^{2}, \overline{\mathbb{C P}}^{2}, S^{1} \times S^{3}, L_{n}$ and $L_{n}^{\prime}(n \geq 2$, integer) (for $L_{n}$ and $L_{n}^{\prime}$, see $\S 4$ ).

Unfortunately, this connected sum decomposition is not unique. For example, $\mathbb{C P}^{2} \#\left(S^{2} \times S^{2}\right)=\mathbb{C P}^{2} \# \overline{\mathbb{C P}}^{2} \# \mathbb{C P}^{2}$. So Pao defined a normal form called a normal decomposition, of this connected sum decomposition. He proved the following topological classification theorem: Every 4-dimensional orientable closed manifold with a $T^{2}$-action has a unique normal decomposition if its orbit space has a fixed point.

Because the classification of closed orientable 4-manifolds which admit an effective $T^{2}$-action is almost complete, it is natural to ask whether there exist similar results for nonorientable 4-manifolds. Furthermore, nonorientable 4-manifolds have not been studied as extensively as the orientable ones, and there are not many concrete examples of them in the literature. By studying nonorientable 4-manifolds with a $T^{2}$-action we are able to give concrete realizations by means of orbit spaces and orbit structures. The orbit space is a 2-manifold with boundary to which are attached certain "weights" which enable us to reconstruct the 4-manifold. While this is not a cell decomposition, it behaves like one in that the manifold is divided into nice pieces which we can topologically identify. We then compute the corresponding attaching maps and attempt to topologically identify and classify the resulting 4-manifolds.

First, we study the problem of classifying nonorientable 4-dimensional manifolds up to equivariant diffeomorphism. in $\S 2$. The key to this is the existence of a cross-section (2.6). This enables us to reconstruct the 4-manifold in certain basic cases from the weighted orbit space. This is a 2-manifold with boundary weighted by the orbit invariants of the action.

In $\S 3$, we investigate simple $T^{2}$-manifolds whose orbit spaces are of the following type. (See Figure 2.) They admit cross-sections to the orbit mapping. The topological identification of these $T^{2}$-manifolds proves to be very difficult. We use well-known invariants to distinguish all but a pair of them. The final equivariantly inequivalent pair cannot be distinguished by any of the previously 


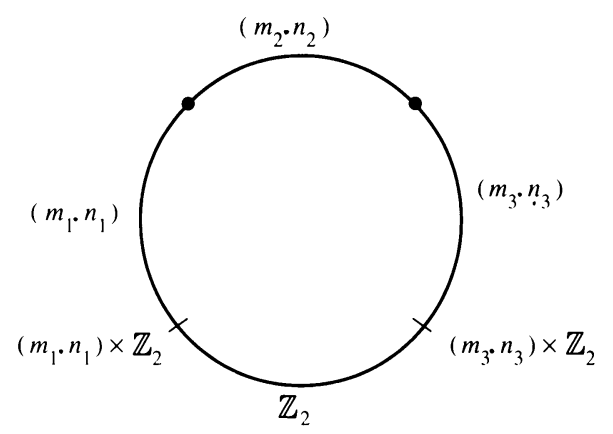

FIGURE 2

known homotopy type invariants. However, by using a new invariant, due to S. Kojima (see [KKR]), constructed for the purpose of distinguishing these manifolds, we are able to show they are not homotopy equivalent. In general, this invariant is hard to compute, but, because we have such a nice description of our manifolds, we are able to give explicit geometric computations.

In $\S 4$, we turn to the problem of topologically identifying the nonorientable closed $T^{2}$-manifolds which admit fixed points, but no $\mathbb{Z}_{2} \times \mathbb{Z}_{2}$ stabilizers on the boundary of their orbit spaces. We show that, by adding connected sums of $\mathbb{C P}^{2}$ 's, the resulting manifold can be decomposed into a connected sum of eight basic manifolds and two families of $T^{2}$-manifolds. We also show that this decomposition is far from unique.

\section{THE EXISTENCE OF A CROSS-SECTION AND THE EQUIVARIANT CLASSIFICATION THEOREM}

Notation and definition. All manifolds are nonorientable 4-dimensional smooth manifolds, unless specified otherwise. $I=\left[\begin{array}{ll}0 & 1\end{array}\right] . S^{1}=$ the set of all complex numbers whose absolute value is $1 . \quad R=$ all real numbers. $\mathbb{C}=$ all complex number $\mathbb{C}^{2}=$ the 2-dimensional complex plane. $R^{2}=2$-dimensional Euclidean space. $T^{2}=S^{1} \times S^{1}$ (2-dimensional torus).

(2.1) Definition. Let $f$ be a function from $R^{2}$ to $\mathbb{C}^{2}$ defined by $f(x . y)=$ $(\exp (2 \pi i x), \exp (2 \pi i y))$, where $\exp (2 \pi i t)=\cos 2 \pi t+l \sin 2 \pi t, t$ a real number. Given relatively prime integers $m, n$, we define the image of the straight line $m x+n y=0$ in $R^{2}$ under $f$ to be $(m . n)$.

(2.2) Definition. A $T^{2}$-action on $M$ is effective if $g x=x$, for all $x$ in $M$, implies $g=e$ (i.e. identity) in $T^{2}$. In this case, we call $M$ a $T^{2}$-manifold. We denote the quotient space (or orbit space) by $M^{*}$ (i.e. $M / T^{2}$ ), and the natural projection from $M$ to $M^{*}$ is $\pi$.

(2.3) Definition. Weighted orbit space. Let $M$ be a $T^{2}$-manifold. Using the slice theorem, we find that $M^{*}$ is a 2-dimensional manifold. By assigning the stabilizer subgroups of $T^{2}$ to orbits in $M^{*}$ as "weights", we may speak of $M^{*}$ as the weighted orbit space (briefly, orbit space). For example, 


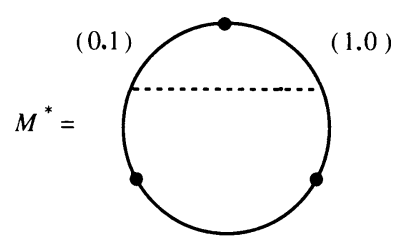

(1.1)

is a weighted disk. The weight at each interior point is the identity, while we have divided up the boundary into three arcs. The three end points of the arcs correspond to fixed points and the interior of the arcs correspond to orbits whose stabilizers are $(0.1),(1.1),(1.0)$ (see [OR, I] for more details). According to [OR, I], the preimage of the dotted line is an invariant $S^{3}$, the portion of the disk above the line is an invariant 4-dimensional disk and the portion of the disk below the line is an invariant disk fiber bundle over $S^{2}$ with a structure group $S^{1}$ in $M$. Therefore, we can see that $M$ is $\mathbb{C P}^{2}$.

We begin with some preliminary steps for the proof of a cross-section theorem, which will be crucial in the Equivariant Classification Theorem (2.7).

(2.4) Lemma. Let $\pi: M \rightarrow M^{*}$ be as above. Suppose that $M^{*}(=I \times I)$ is given as below:

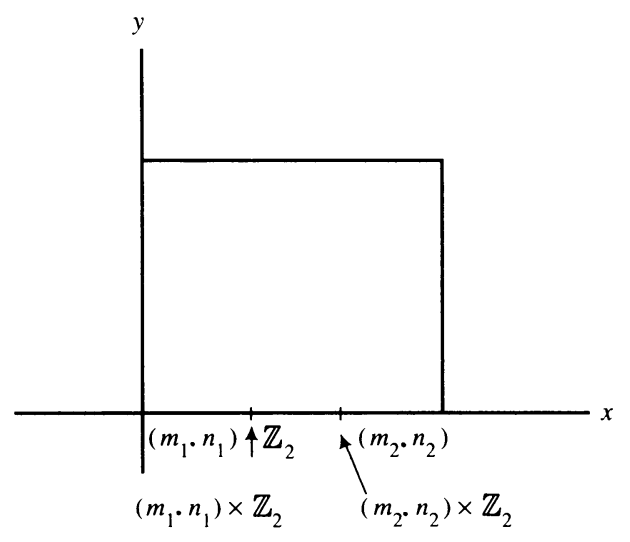

More precisely, on the set $\{(x, 0): 0 \leq x<1 / 3\}$, the stabilizer is $\left(m_{1} . n_{1}\right)$. On the set $\{(x, 0): 1 / 3<x<2 / 3\}$, the stabilizer is a $\mathbb{Z}_{2}$ subgroup of $T^{2}$. On the set $\{(x, 0): 2 / 3<x<1\}$, the stabilizer is $\left(m_{2} . n_{2}\right)$, at $(1 / 3,0)$, the stabilizer is $\left(m_{1}, n_{1}\right) \times \mathbb{Z}_{2}$, at $(2 / 3,0)$, the stabilizer is $\left(m_{2} . n_{2}\right) \times \mathbb{Z}_{2}$. Otherwise the stabilizer is trivial. Then $\pi$ has a cross-section (i.e. there exists a map $\chi$ such that $\pi \circ \chi=\mathrm{Id})$. Moreover, any cross-section $\chi$ on $I \times\{1\} \cup\{0\} \times I \cup\{1\} \times I=A$ may be extended to a cross-section over $M^{*}$. Note that $\mathbb{Z}_{2}$ is not contained in the circle subgroups.

Proof. Since $M^{*}$ has a $\mathbb{Z}_{2}$ stabilizer on the boundary, by considering the slice representation, $M$ is nonorientable. Let $\widetilde{M}$ be the orientable double covering of $M$. Then, we have an induced $T^{2}$-action on $M$ which commutes with the 
regular covering transformations (cf. [B, p. 66]). We have the induced $\mathbb{Z}_{2}$-action on $\widetilde{M}^{*}$ and the natural projection from $\widetilde{M}^{*}$ to $M^{*}$. In particular, $\widetilde{M}^{*}=I \times I$ is given as below:

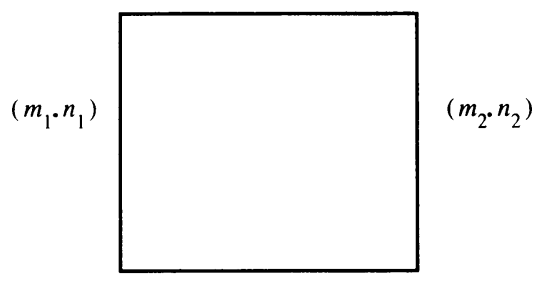

where $A_{1}=I \times\{1\}$. The induced $\mathbb{Z}_{2}$-action on $\widetilde{M}^{*}$ acts a rotation by 180 degrees or a reflection. Since $M$ is nonorientable, it must act as a reflection. Therefore, there exists a cross-section $\chi_{1}$ from $M^{*}$ to $\widetilde{M}^{*}$. By [OR, I], there exists a cross-section $\chi_{2}$ from $\widetilde{M}^{*}$ to $\widetilde{M}$ and $\widetilde{M}=L \times I$ where $L$ is a lens space, whose exact form depends on $\left(m_{1} . n_{1}\right)$ and $\left(m_{2} . n_{2}\right)$.

Consider the map $\rho \circ \chi_{2} \circ \chi_{1}$ from $M^{*}$ to $M$, where $\rho$ is the natural projection from $\widetilde{M}$ to $M$. Note that $\rho^{*} \circ \tilde{\pi}=\pi \circ \rho$ where $\rho^{*}$ is the natural projection from $\widetilde{M}^{*}$ to $M^{*}$, and $\tilde{\pi}$ is the natural projection from $\widetilde{M}$ to $\widetilde{M}^{*}$. Then $\pi \circ \rho \circ \chi_{2} \circ \chi_{1}=\rho^{*} \circ \tilde{\pi} \circ \chi_{2} \circ \chi_{1}=\rho^{*} \circ \operatorname{Id}_{\widetilde{M}^{*}} \circ \chi_{1}=\rho \circ \chi_{1}=\operatorname{Id}_{M^{*}}$.

So $\rho \circ \chi_{2} \circ \chi_{1}$ is a cross-section to the orbit map $\pi$. Moreover, given a cross-section $\chi$ on $A$, by [OR, I], $\chi(A)$ is a lens space $L$ in $M$.

Since $L$ is orientable and $\rho: \widetilde{M} \rightarrow M$ is a double covering, $\rho^{-1}(\chi(A))$ is the disjoint union of two lens spaces, i.e. $\rho^{-1}(\chi(A))=L \times\{0\} \cup L \times\{1\}$. We may assume that $\chi_{2}\left(A_{1}\right)=L \times\{0\}$. Then $\chi_{1} \circ \chi(A)=L \times\{0\}$. Since $\rho^{*} \circ\left(\tilde{\pi} \circ \chi_{1} \circ \chi\right)=\pi \circ \rho \circ \chi_{1} \circ \chi=\pi \circ \operatorname{Id}_{M} \circ \chi=\operatorname{Id}_{M^{*}} \tilde{\pi} \circ \chi_{1} \circ \chi$ is a cross-section from $A$ to $\widetilde{M}^{*}$ and $\tilde{\pi} \circ \chi_{1} \circ \chi(A)=A_{1}$.

Let $\tilde{\chi}=\tilde{\pi} \circ \chi_{1} \circ \chi$. We can extend $\tilde{\chi}$ to $M^{*}$ in an obvious way. So $\rho \circ \chi_{2} \circ \tilde{\chi}$ is an extension of $\chi$.

(2.5) Lemma. Let $\pi: M \rightarrow M^{*}$ be as above. Suppose $M^{*}=I \times I$ is shown as below:

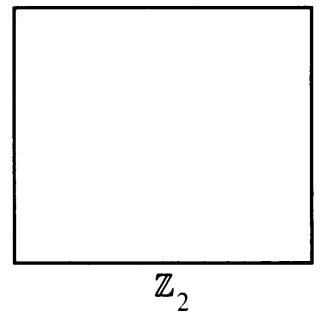

More precisely, the stabilizer is $\mathbb{Z}_{2}$ on the $I \times\{0\}$, and elsewhere it is the identity. Then there exists a cross-section. Moreover, a given cross-section $\chi$ on $I \times\{1\} \cup\{0\} \times I \cup\{1\} \times I$ may be extended to $M^{*}$.

Proof. This is verified by a slight modification of Lemma (2.4).

Now we are ready to state and prove the existence of a cross-section in more general cases. 
(2.6) Proposition. Let $M$ be closed. Suppose $M^{*}$ has a fixed point, has no $\mathbb{Z}_{2} \times \mathbb{Z}_{2}$ stabilizer on the boundary and no finite stabilizer in the interior. Then there exists a cross-section to the orbit map.

Proof. As in Case 3 of Theorem 1.10 of [OR, I], consider a closed annular neighborhood of the boundary components. Denote their union by $U^{*}$. Note that, on $Y^{*}=M^{*}-U^{*}, \pi^{-1}\left(Y^{*}\right) \rightarrow Y^{*}$ is a $T^{2}$-principal bundle. By the classification of 2-dimensional manifolds with boundaries, $Y^{*}$ is homotopy equivalent to $\bigvee S^{1}$ (a wedge product of $S^{1}$ 's).

Since $H^{2}\left(\bigvee S^{1}, \mathbb{Z} \times \mathbb{Z}\right)=H^{2}\left(Y^{*}, \mathbb{Z} \times \mathbb{Z}\right)=0, \pi^{-1}\left(Y^{*}\right)=Y^{*} \times T^{2}$. This we may construct a cross-section over $Y^{*}$ and, by applying Lemmas 1.6, 1.7, 1.8 of [OR, I] and previous lemmas, $(2.4,2.5)$, we get a global cross-section on $M^{*}$.

(2.7) Theorem (Equivariant Classification Theorem). Let $M_{1}, M_{2}$ be closed manifolds. Suppose that both $M_{1}^{*}$ and $M_{2}^{*}$ have a fixed point but have no $\mathbb{Z}_{2} \times \mathbb{Z}_{2}$ stabilizer on the boundaries. Then there exists a weight preserving diffeomorphism between $M_{1}^{*}$ and $M_{2}^{*}$, if and only if there exists an equivariant diffeomorphism between $M_{1}$ and $M_{2}$.

Remark. By the slice theorem, if there is an orbit in the interior of an orbit space whose stabilizer is nontrivial (i,e. other than identity), then the stabilizer is finite.

We can choose a closed disk in the interior of the orbit space. The preimage of the closed disk and the action is topologically equivalent to $\left(T^{2}, T^{2} \times D^{2} / \mathbb{Z}_{\alpha}\right)$. Where $\mathbb{Z}_{\alpha}$ is the finite cyclic stabilizer subgroup of $T^{2}$, and the action of $\mathbb{Z}_{\alpha}$ on $T^{2}$ and $D^{2}$ is given as follows

$$
\begin{gathered}
\lambda \times z \rightarrow \lambda^{\nu} z, \quad|z| \leq 1, \quad z \text { in } D^{2}, \\
\lambda \times\left(z_{1}, z_{2}\right) \rightarrow\left(z_{1} \lambda^{\gamma_{1}}, z_{2} \lambda^{\gamma_{2}}\right), \quad\left(z_{1}, z_{2}\right) \quad \text { in } T^{2},
\end{gathered}
$$

with $0<\nu<\alpha, \alpha$ and $\nu$ are relatively prime, $\lambda=\exp (2 \pi l / \alpha)$ and $0 \leq \gamma_{1}<$ $\alpha, 0 \leq \gamma_{2}<\alpha . T^{2} \times D^{2} / \mathbb{Z}_{\alpha}$ means each point $\left(\left(z_{1}, z_{2}\right), z\right)$ of $T^{2} \times D^{2}$ is identified with $\left(\lambda\left(z_{1}, z_{2}\right), \lambda^{-1} z\right)$. The $T^{2}$-action on $T^{2} \times D^{2} / \mathbb{Z}_{\alpha}$ is on the first coordinate by natural multiplication.

Proof. The argument for this theorem is really the same as Theorem 1.2 of [OR, I]. First, if $M_{1}$ and $M_{2}$ are equivariantly diffeomorphic, then this diffeomorphism induces a weight preserving map on the orbit spaces.

On the other hand, given such a diffeomorphism $h: M_{1}^{*} \rightarrow M_{2}^{*}$, we shall construct an equivariant diffeomorphism from $M_{1}$ to $M_{2}$. Choose closed 2disks $D$ about each $x$ whose stabilizer is finite in the interior. They are mapped by $h$ onto corresponding disks about $h\left(x_{k}^{*}\right)$. We can assume that $x_{k}^{*}$ is in the interior of $D_{k}^{*}$ which is in the interior of $M_{1}^{*}$, and that there is no other point in $D_{k}^{*}$ whose stabilizer is nontrivial. If we let $M_{1.1}^{*}=M_{1}^{*}-\bigcup_{k=1}^{n} D_{k}^{*}$ and $M_{2.1}^{*}=$ $M_{2}^{*}-\bigcup_{k=1}^{n} h\left(\stackrel{\circ}{D}_{k}^{*}\right)$, where $\stackrel{\circ}{D}_{k}^{*}$ is the interior of $D_{k}^{*}$, then $h_{1}: M_{1.1}^{*} \rightarrow M_{2.1}^{*}$, the restriction of $h$, is a weight preserving diffeomorphism.

Furthermore, as there are no finite stabilizers except the identity in the interior of $M_{1.1}$, any cross-section of the orbit map over $\bigcup_{k=1}^{n} \partial D_{k}^{*}$ can be extended to all of $M_{1.1}^{*}$ by Lemma (1.10) of [OR, I]. Thus it is easy to find an equivariant diffeomorphism $H: M_{1.1} \rightarrow M_{2.1}$. 
To each $M_{l \cdot 1},(l=1,2)$, we must attach the $\left(T^{2}, T^{2} \times D_{k} / \mathbb{Z}_{\alpha, k}\right)$ equivariantly, where $\mathbb{Z}_{\alpha, k}$ means $\mathbb{Z}_{\alpha}$ acting on $D_{k}$. We already have a cross section over each $\partial D_{k}^{*}$. We may attach $\left(T^{2}, T^{2} \times D_{k} / \mathbb{Z}_{\alpha, k}\right)$ up to equivariant diffeomorphism in only one way (up to equivariant isotopy) and thereby extend $H$ to all of $M_{1}$. This completes the proof.

\section{SPecial CASEs}

Notation and definition. Let $R^{3}$ be 3-dimensional Euclidean space. Let $\nu$ denote a vector $R^{3} . S^{2}=\left\{(x, y, z)\right.$ in $\left.R^{3}: x^{2}+y^{2}+z^{2}=1\right\}=\{\nu:|\nu|=$ $1\}$. Since we may identify $R^{3}$ with $\mathbb{C} \times R, S^{2}$ may be expressed as follows: $S^{2}=\left\{(\rho \exp (2 \pi l \theta), z): \rho \geq 0, \rho^{2}+z^{2}=1\right\} . R P^{2}$ is real projective 2space. We will use $[\nu]$ or $[(\rho \exp (2 \pi l \theta), z)]$ respectively, to denote the point in $R P^{2}$ which is the image of $\nu$ or $(\rho \exp (2 \pi l \theta), z)$ in $S^{2}$ under the natural projection from $S^{2}$ to $R P^{2}$. $\mathbb{C P}^{2}$ is complex projective 2-space. $S^{4}=$ the unit 4-sphere and $R P^{4}=$ the real projective 4-space. $D^{2}$ is the unit disk in $R^{2}$, i.e. $\left\{(x \cdot y): x^{2}+y^{2} \leq 1\right\}=\{r \exp (2 \pi l \phi): 0 \leq r \leq 1, \phi \in R\} . S^{1} \times S^{2} / \simeq$ is the nontrivial $S^{2}$ bundle over the circle (i.e. the 3-dimensional nonorientable handle. Here $\simeq$ means every $(\alpha, \nu)$ in $S^{1} \times S^{2}$ is identified with $(-\alpha,-\nu)$, $\alpha \in \mathbb{C}$.

In this section, we shall first describe some special orbit spaces, then construct manifolds with $T^{2}$-actions, whose orbit spaces are the given ones. Then we will give the topological classification of these manifolds. (In fact, two distinct such manifolds are not even homotopy equivalent).

Now suppose that we have orbit spaces as shown in Figure 3 where $\mathbb{Z}_{2}$ is a subgroup of order 2 in $T^{2}$.

Recall from [OR, I] that, as the $T^{2}$-action is smooth and effective, determinants

$$
\left|\begin{array}{ll}
m_{1} & n_{1} \\
m_{2} & n_{2}
\end{array}\right| \text { and }\left|\begin{array}{ll}
m_{2} & n_{2} \\
m_{3} & n_{3}
\end{array}\right|
$$

must be 1 or -1 .

By using an automorphism of $T^{2}$ (i.e. by reparametrizing $T^{2}$ ), we may assume that $M^{*}$ is one of the following four types, where $n, m, n^{\prime}, m^{\prime}$ are

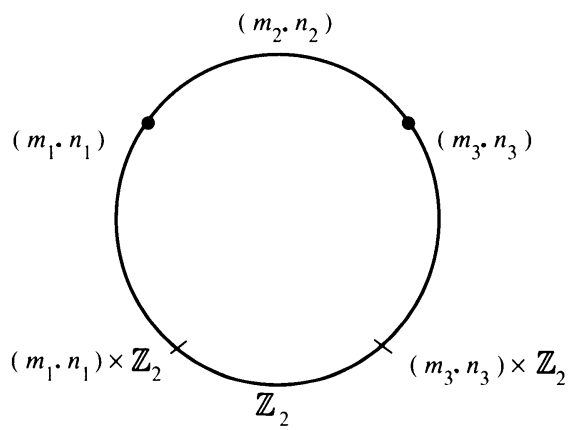

FIGURE 3 
integers. In type II and type IV, note that $m, m^{\prime}$ must be even, because effectiveness implies $(1 . \mathrm{m}),\left(-1 . \mathrm{m}^{\prime}\right)$ cannot contain the subgroup $\langle-1 \times-1\rangle$ of order 2 generated by $-1 \times-1 \in S^{1} \times S^{1}$.
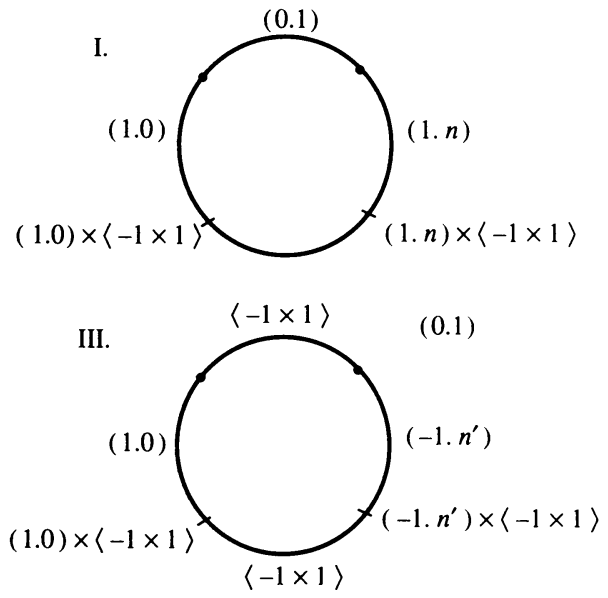
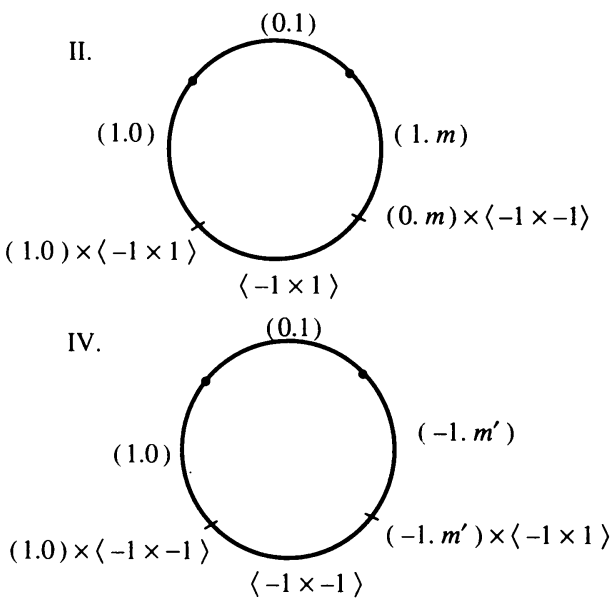

(3.2) Theorem. Let $M^{*}$ be of type I or type III. Then $M$ is diffeomorphic to $R P^{2} \times S^{2}$ or $\mathbb{C P}^{2} \# R P^{4}$ according as $n$ is even or odd.

Remark. If $M$ is a nonorientable manifold and $X$ is oriented, then $M \# X$ is diffeomorphic to $M \# \bar{X}$, where $\bar{X}$ denotes $X$ with the reverse orientation. So $\mathbb{C P}^{2} \# R P^{4}$ is diffeomorphic to $\overline{\mathbb{C P}}^{2} \# R P^{4}$ (see $\left.[\mathrm{H}]\right)$.

To prove (3.2), we need the following lemma.

(3.3) Lemma. Let $f_{n}$ be a diffeomorphism from $S^{1} \times R P^{2}$ to $S^{1} \times R P^{2}$ defined as follows:

$$
\begin{aligned}
& (\exp (2 \pi l \phi),[(r \exp (2 \pi l \theta), z)]) \\
& \quad \rightarrow(\exp (2 \pi l \phi),[(r \exp 2 \pi l(\theta+n \phi), z)]) .
\end{aligned}
$$

Then, if $n$ is even, $f_{n}$ can be extended to $D^{2} \times R P^{2}$ as a self-diffeomorphism.

Proof. $f_{n}$ may be written in the following way:

$$
(\exp (2 \pi l \phi),[\nu]) \rightarrow\left(\exp (2 \pi l \phi),\left(\begin{array}{ccc}
\cos 2 \pi n \phi & \sin 2 \pi n \phi & 0 \\
-\sin 2 \pi n \phi & \cos 2 \pi n \phi & 0 \\
0 & 0 & 1
\end{array}\right)(\nu)\right)
$$

Then the map $\tilde{f}_{n}$ from $S^{1}$ to $S \mathrm{O}(3)$ defined by

$$
\exp (2 \pi l \phi) \rightarrow\left(\begin{array}{ccc}
\cos 2 \pi n \phi & \sin 2 \pi n \phi & 0 \\
-\sin 2 \pi n \phi & \cos 2 \pi n \phi & 0 \\
0 & 0 & 1
\end{array}\right)
$$

is a loop in $S \mathrm{O}(3)$, so $\tilde{f}_{n}$ represents an element in $\Pi_{1}(S O(3))$. Since $n$ is even, $\tilde{f}_{n}$ is homotopic to the trivial map i.e. there exists a homotopy $H_{n}$ from 
$S^{1} \times I$ to $S O(3)$ such that

$$
\begin{aligned}
& H_{n}(\exp (2 \pi l \phi), 1)=\tilde{f}_{n}(\exp (2 \pi l \phi)) \\
& H_{n}(\exp (2 \pi l \phi), 0)=\left(\begin{array}{lll}
1 & 0 & 0 \\
0 & 1 & 0 \\
0 & 0 & 1
\end{array}\right) .
\end{aligned}
$$

Define $F_{n}$ from $D^{2} \times R P^{2}$ to $D^{2} \times R P^{2}$ by

$$
(r \exp (2 \pi l \phi),[\nu]) \rightarrow\left(r \exp (2 \pi l \phi),\left[H_{n}(\exp (2 \pi l \phi), r)[\nu]\right)\right.
$$

Then since $F_{n}(r \exp (2 \pi l \phi),[-\nu])=\left(r \exp (2 \pi l \phi),\left[H_{n}(\exp (2 \pi l \phi), r)(-\nu)\right]\right)=$ $F_{n}(r \exp (2 \pi l \phi),[\nu]), F_{n}$ is a well-defined diffeomorphism. This completes the proof.

Let us return to (3.2).

Proof of Theorem (3.2). First, we will construct a manifold with a $T^{2}$-action whose orbit space is

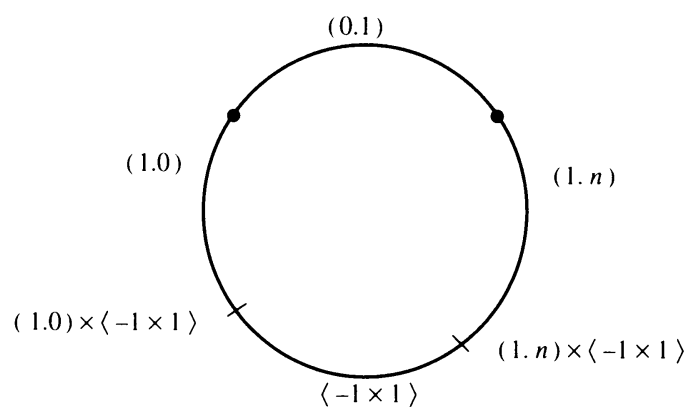

Consider a $T^{2}$-action on $D^{2} \times R P^{2}$ as follows:

$$
\begin{gathered}
T^{2} \times D^{2} \times R P^{2} \rightarrow D^{2} \times R P^{2}, \\
(\exp (2 \pi l \alpha), \exp (2 \pi l \beta))(r \exp (2 \pi l \phi),[\rho \exp (2 \pi l \theta), z]) \\
\rightarrow(r \exp 2 \pi l(\phi+\beta),[\rho \exp 2 \pi l(\alpha+n \beta+\theta), z]) .
\end{gathered}
$$

Then,

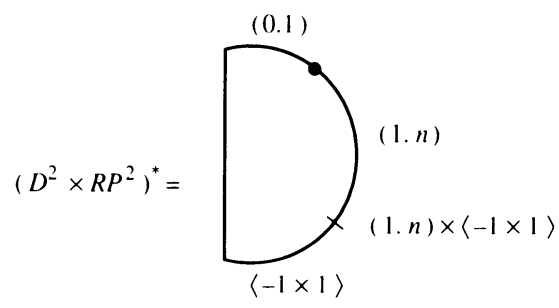


In particular, when $n=0$,

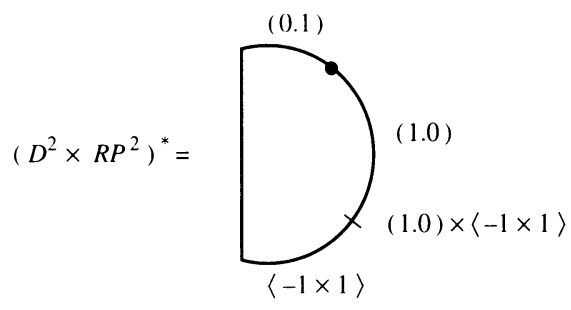

If we glue two copies of $D^{2} \times R P^{2}$ along the boundary by using the map $f_{n}$ in (3.3), we get a 4-manifold $D^{2} \times R P^{2} \cup_{f_{n}} D^{2} \times R P^{2}$. On the first copy, we give the action above when $n=0$, and on the second copy, we give the action for general $n$. Then we can see that $f_{n}$ is an $T^{2}$-equivariant diffeomorphism from $\partial D^{2} \times S^{2}$ to $\partial D^{2} \times S^{2}$.

Thus we have an 4-manifold $D^{2} \times R P^{2} \cup_{f_{n}} D^{2} \times R P^{2}$ with a $T^{2}$-action whose orbit space is

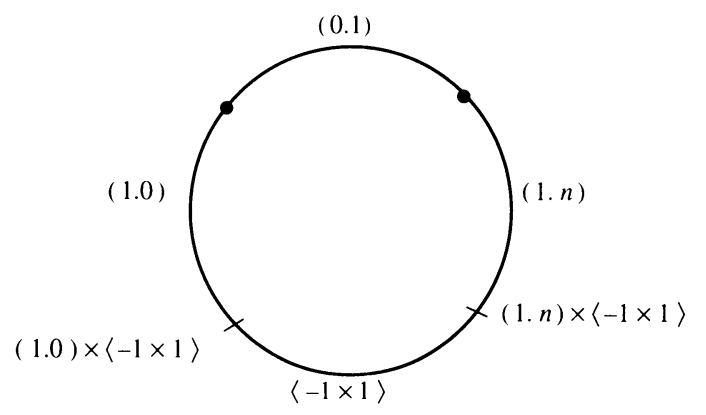

Now we can complete the proof of the theorem as follows.

(i) $n$ is even. Define a map $\operatorname{Id} * F_{n}$ from $D^{2} \times R P^{2} \cup_{\text {id }} D^{2} \times R P^{2}$ to $D^{2} \times$ $R P^{2} \cup_{f_{n}} D^{2} \times R P^{2}$ as follows:

On the first copy of $D^{2} \times R P^{2}$,

$$
(r \exp (2 \pi l \phi),[\nu]) \rightarrow(r \exp (2 \pi l \phi),[\nu])
$$

on the second copy of $D^{2} \times R P^{2}$,

$$
(r \exp (2 \pi l \phi),[\nu]) \rightarrow\left(r \exp (2 \pi l \phi),\left[H_{n}(\exp (2 \pi l \phi), r)(\nu)\right]\right)
$$

Then Id $* F_{n}$ is a well-defined diffeomorphism.

(ii) $n$ is odd. $n-1$ is even, so we can apply (3.3). Define $\operatorname{Id} * F_{n-1}$, as in the case (i), from $D^{2} \times R P^{2} \cup_{f_{1}} D^{2} \times R P^{2}$ to $D^{2} \times R P^{2} \cup_{f_{n}} D^{2} \times R P^{2}$. Then Id $* F_{n-1}$ is a well-defined diffeomorphism.

If we put $M_{0}=D^{2} \times R P^{2} \cup_{\text {id }} D^{2} \times R P^{2}$, and $M_{1}=D^{2} \times R P^{2} \cup_{f_{1}} D^{2} \times R P^{2}$, $M_{0}=S^{2} \times R P^{2}$, and we see that $M_{1}$ is the nontrivial $R P^{2}$-fiber bundle over $S^{2}$ with the structure group $S^{1}$. Furthermore, consider the dotted line in $M_{1}^{*}$, 


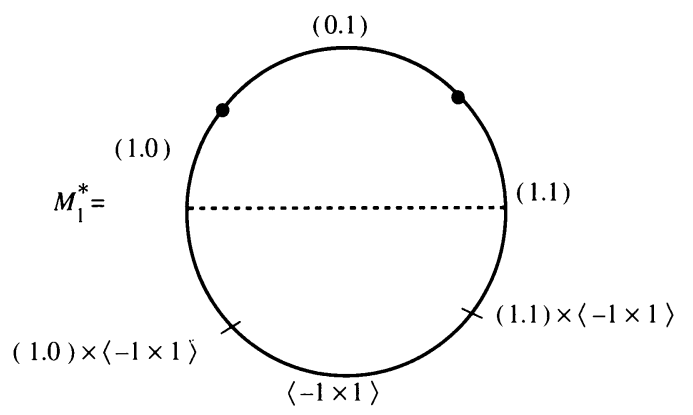

The preimage in $M_{1}$ of the line is a $T^{2}$-invariant $S^{3}$. If we cut $M_{1}$ along that $S^{3}$, we have $M_{1.1}$ and $M_{1.2}$ whose orbit spaces are
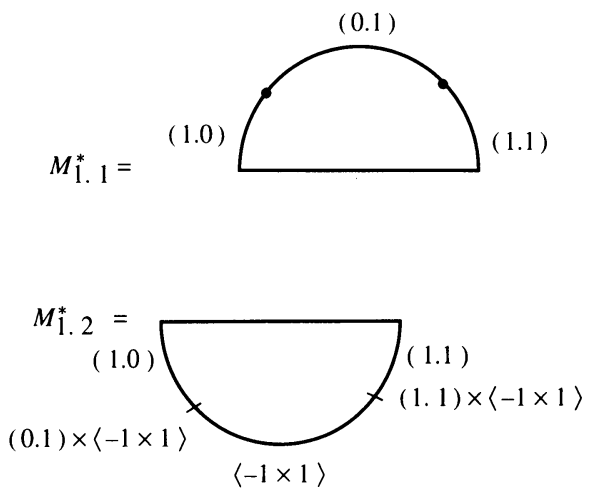

By adding two copies of

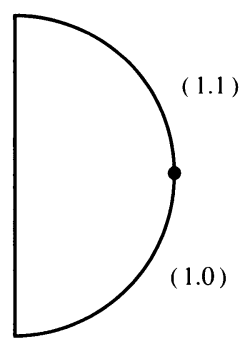

whose total space is a 4-dimensional disk, to $M_{1.1}^{*}$ and $M_{1.2}^{*}$, we have
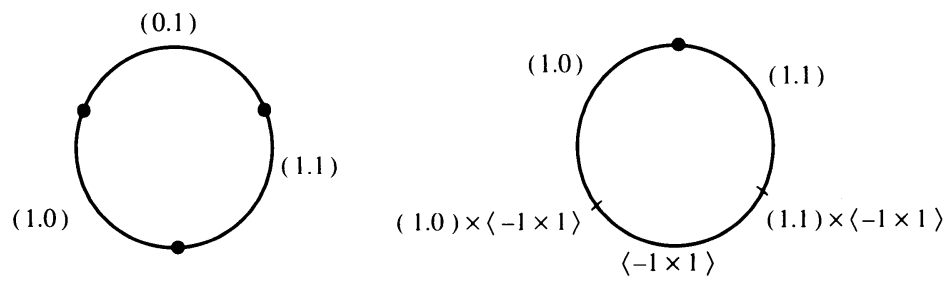
In other words, to total spaces $M_{1.1}$ and $M_{1.2}$, we have to attach 4-dimensional disks along the boundaries equivariantly.

This procedure implies that $M_{1}$ is a connected sum of two manifolds. By [OR, I], a manifold with the first orbit space is diffeomorphic to $\mathbb{C P}^{2}$. For the second orbit space, we give a $T_{2}$-action on $R P^{4}$ as follows:

Since

$$
\left(\begin{array}{rrrr}
-1 & 0 & 0 & 0 \\
0 & -1 & 0 & 0 \\
0 & 0 & -1 & 0 \\
0 & 0 & 0 & -1
\end{array}\right)
$$

commutes with

$$
\left(\begin{array}{ccccc}
\cos 2 \pi \theta & \sin 2 \pi \theta & 0 & 0 & 0 \\
-\sin 2 \pi \theta & \cos 2 \pi \theta & 0 & 0 & 0 \\
0 & 0 & \cos 2 \pi \phi & \sin 2 \pi \phi & 0 \\
0 & 0 & -\sin 2 \pi \phi & \cos 2 \pi \phi & 0 \\
0 & 0 & 0 & 0 & 1
\end{array}\right)
$$

we can give a $\mathbb{Z}_{2} \times T^{2}$-action on $S^{4}$ naturally and also give a $T^{2}$-action on $R P^{4}$, there is only one orbit space up to automorphism of $T^{2}$. Thus the manifold whose orbit space is

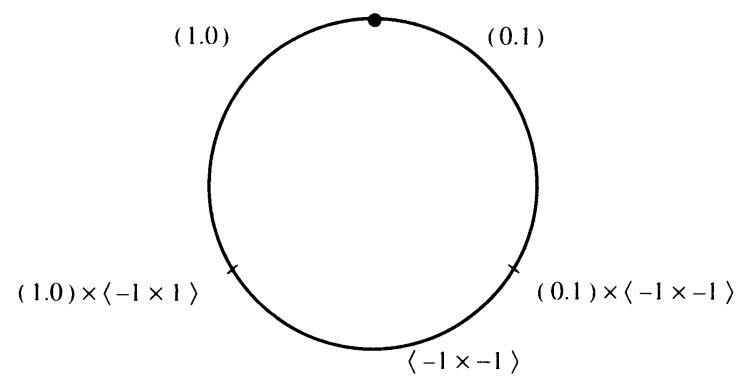

is $R P^{4}$. We conclude that any manifold $M$ of type $\mathrm{I}$ is diffeomorphic to $R P^{4} \# \mathbb{C P}^{2}$.

In the same way, by giving appropriate $T^{2}$-actions on two copies of $D^{2} \times R P^{2}$ and gluing the copies along the boundaries, we can construct a manifold whose orbit space is of type III as follows:

$$
\begin{gathered}
T^{2} \times D^{2} \times R P^{2} \rightarrow D^{2} \times R P^{2}, \\
(\exp (2 \pi l \alpha), \exp (2 \pi l \beta)) \times(r \exp (2 \pi l \phi),[\rho \exp (2 \pi l \theta), z]) \\
\rightarrow\left(r \exp 2 \pi l(\phi+\beta),\left[\rho \exp 2 \pi l\left(-\alpha+n^{\prime} \beta+\theta\right), z\right]\right), \\
T^{2} \times D^{2} \times R P^{2} \rightarrow D^{2} \times R P^{2}, \\
(\exp (2 \pi l \alpha), \exp (2 \pi l \beta)) \times(r \exp (2 \pi l \phi),[\rho \exp (2 \pi l \theta), z]) \\
\rightarrow(r \exp 2 \pi l(\phi+\beta),[\rho \exp 2 \pi l(\alpha+\theta), z]) .
\end{gathered}
$$

The orbit spaces are as follows: 

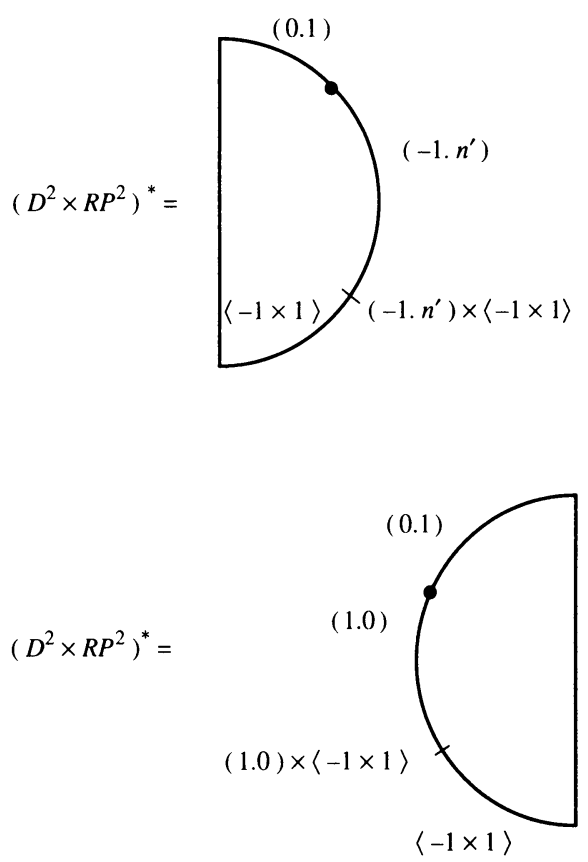

Let $f_{n}^{\prime}$ be a self-diffeomorphism of $\partial D^{2} \times R P^{2}$ defined by

$$
(\exp (2 \pi l \phi),[\rho \exp (2 \pi l \theta), z]) \rightarrow\left(\exp (2 \pi l \phi),\left[\rho \exp 2 \pi l\left(-\theta+n^{\prime} \phi\right), z\right]\right)
$$

We can see that the map is $T^{2}$-equivariant with respect to the above two actions. Since the set of self-diffeomorphisms of $R P^{2}$ is path-connected, the map, when $n^{\prime}=0$, is isotopic to identity. So we can apply the procedure in type I. This completes the proof.

Remark. For the above two spaces, by considering double coverings with their induced $T^{2}$-actions, we can see that their orbit spaces are

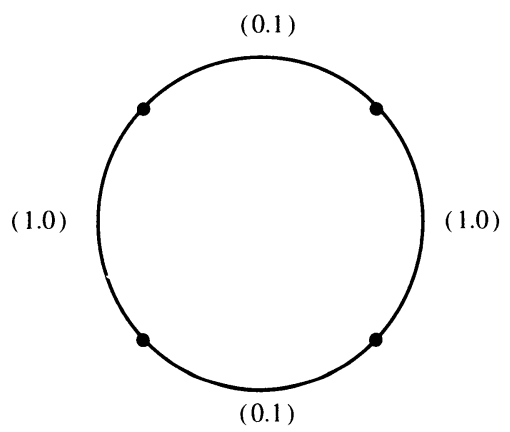

and 


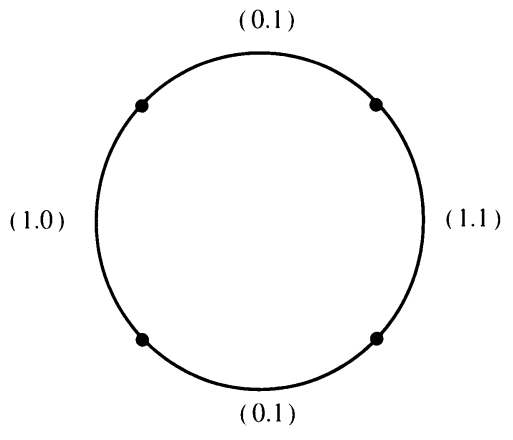

respectively.

So $R P^{4} \# \mathbb{C P}^{2}$ has $\mathbb{C P}^{2} \# \overline{\mathbb{C P}}^{2}$ as its orientable double covering and $S^{2} \times R P^{2}$ has $S^{2} \times S^{2}$ as its orientable double covering by [OR, I]. Therefore $R P^{4} \# \mathbb{C P}^{2}$ is not homotopy equivalent to $S^{2} \times R P^{2}$.

(3.4) Theorem. Let $M^{*}$ be of type II or type IV. Then $M$ is diffeomorphic to $S^{2} \times S^{2} / \simeq$ or $D^{2} \times S^{2} / \simeq \cup_{g_{2}} D^{2} \times S^{2} / \simeq$.

In the first case, the equivalence relation $\simeq$ means $((\rho \exp (2 \pi l \phi), z, \nu)$ is identified with $((-\rho \exp (2 \pi i \phi), z),-\nu)$. In the second case, $\simeq$ means $(r \exp (2 \pi l \phi), \nu)$ is identified with $(-r \exp (2 \pi l \phi),-\nu)$. The gluing map $g_{2}$ of two $D^{2} \times S^{2} / \simeq$ from $\partial D^{2} \times S^{2} / \simeq$ to itself defined by

$$
[(\exp (2 \pi l \phi), \nu)] \rightarrow\left[\left(\exp (2 \pi l \phi), \tilde{f}_{2}(\exp (2 \pi l \phi))(\nu)\right)\right],
$$

$\tilde{f}_{2}$ is the map in (3.3), according as $m=4 j$ or $m=4 j+2, j$ integer. As in (3.2), we need the following lemma.

(3.5) Lemma. Let $g_{m}$ be a self-diffeomorphism from $S^{1} \times S^{2} / \simeq$ to itself, defined by

$$
[(\exp (2 \pi l \phi), \nu)] \rightarrow\left[\left(\exp (2 \pi l \phi), \tilde{f}_{m}(\exp (2 \pi l \phi))(\nu)\right)\right]
$$

Then, if $m=4 j, g_{m}$ can be extended to $D^{2} \times S^{2} / \simeq$ where $\tilde{f}_{m}$ is the map in (3.3).

Proof. Recall from (3.3) that the map $\tilde{f}_{m}: S^{1} \rightarrow S O(3)$ is given by

$$
\begin{gathered}
\exp (2 \pi l \phi) \rightarrow\left(\begin{array}{ccc}
\cos 2 \pi m \phi & \sin 2 \pi m \phi & 0 \\
-\sin 2 \pi m \phi & \cos 2 \pi m \phi & 0 \\
0 & 0 & 1
\end{array}\right) \\
H_{m}: S^{1} \times I \rightarrow S O(3) \\
H_{m}(\exp (2 \pi l \phi), 1)=\tilde{f}_{m}(\exp (2 \pi l \phi)) \\
H_{m}(\exp (2 \pi l \phi), 0)=\left(\begin{array}{lll}
1 & 0 & 0 \\
0 & 1 & 0 \\
0 & 0 & 1
\end{array}\right)
\end{gathered}
$$

Define a map $\widetilde{G}_{2 j}$ from $D^{2} \times S^{2} / \simeq$ to itself by

$$
[(r \exp (2 \pi l \phi), \nu)] \rightarrow\left[\left(r \exp (2 \pi l \phi), H_{2 j}(\exp (2 \pi l \phi, r))(\nu)\right)\right] .
$$


Then, since $\tilde{f}_{2 j}(\exp (2 \pi l \phi))=\tilde{f}_{4 j}(\exp (2 \pi l \phi)), \widetilde{G}_{2 j}$ is a well-defined diffeomorphism. This completes the proof.

Proof of (3.4). As in the proof of (3.2), we shall construct a manifold whose orbit space is of type II, and get the same results on type IV. Consider $T^{2}$-actions on $D^{2} \times S^{2} / \simeq$ as follows:

$$
\begin{gathered}
T^{2} \times D^{2} \times S^{2} / \simeq \rightarrow D^{2} \times S^{2} / \simeq, \\
(\exp (2 \pi l \alpha), \exp (2 \pi l \beta)) \times[(r \exp (2 \pi l \phi),\{\rho \exp (2 \pi l \theta), z\})] \\
\rightarrow[(r \exp 2 \pi l(\phi+\beta),\{\rho \exp 2 \pi l(\theta+\alpha+m \beta), z\})], \\
T^{2} \times D^{2} \times S^{2} / \simeq \rightarrow D^{2} \times S^{2} / \simeq, \\
(\exp (2 \pi l \alpha), \exp (2 \pi l \beta)) \times[(r \exp (2 \pi l \phi),\{\rho \exp (2 \pi l \theta), z\})] \\
\rightarrow[(r \exp 2 \pi l(\phi+\beta),\{\rho \exp 2 \pi l(\theta+\alpha+m \beta), z\})] .
\end{gathered}
$$

Then, its orbit spaces are respectively,

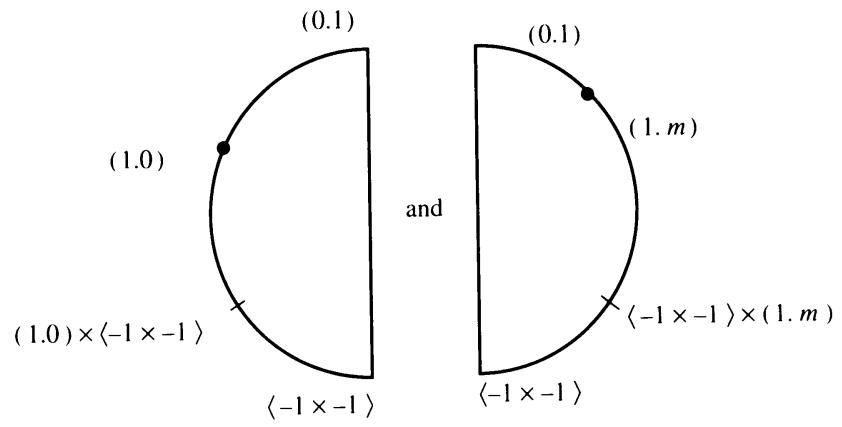

It is easy to check that $g_{m}$ in (3.5) is a $T^{2}$-equivariant (with respect to the above two actions) self-diffeomorphism of $D^{2} \times S^{2} / \simeq$. So by gluing two copies of $D^{2} \times S^{2} / \simeq$ along the boundary through $g_{m}$, we have a $T^{2}$-manifold $D^{2} \times S^{2} / \simeq \cup_{g_{m}} D^{2} \times S^{2} / \simeq$

(i) $m=4 j$. Define an $\operatorname{Id} * \widetilde{G}_{2 j}$ as follows:

$$
D^{2} \times S^{2} / \simeq \cup_{\text {Id }} D^{2} \times S^{2} / \simeq \rightarrow D^{2} \times S^{2} / \simeq \cup_{g_{4 j}} D^{2} \times S^{2} / \simeq
$$

on the first copy of $D^{2} \times S^{2} / \simeq$

$$
[(r \exp (2 \pi l \phi), \nu)] \rightarrow[(r \exp (2 \pi l \phi), \nu)]
$$

on the second copy of $D^{2} \times S^{2} / \simeq$

$$
\left[(r \exp (2 \pi l \phi), \nu] \rightarrow \widetilde{G}_{2 j}([(r \exp (2 \pi l \phi), \nu)]) .\right.
$$

Then, Id $* \widetilde{G}_{2 j}$ is a well-defined diffeomorphism, since

$$
\widetilde{G}_{2 j}[(-r \exp (2 \pi l \phi),-\nu)]=-\widetilde{G}_{2 j}[(r \exp (2 \pi l \phi), \nu)] .
$$

(ii) $m=4 j+2$. We have a well-defined diffeomorphism Id $* \widetilde{G}_{2 j}$, as in the case $(\mathrm{i})$, from $D^{2} \times S^{2} / \simeq \cup_{g_{2}} D^{2} \times S^{2} / \simeq$ to $D^{2} \times S^{2} / \simeq \cup_{g_{4 j+2}} D^{2} \times S^{2} / \simeq$. Note 
that $D^{2} \times S^{2} / \simeq \cup_{\mathrm{id}} D^{2} \times S^{2} / \simeq=S^{2} \times S^{2} / \simeq$. Similarly, for type IV, we can make a construction analogous to type II. This completes the proof.

Remark. $S^{2} \times S^{2} / \simeq$ is an $S^{2}$-bundle over $R P^{2}$ with the structure group $\mathbb{Z}_{2}$. The self-diffeomorphism $g_{2}$ is not diffeotopic to the identity (see [KR]).

(3.6) Theorem. $R P^{2} \times S$ is not homotopy equivalent to $S^{2} \times S^{2} / \simeq$.

Proof. We want to show that all elements in $H^{2}\left(S^{2} \times R P^{2}, \mathbb{Z}_{2}\right)$ have zero square, i.e. for every $l$ in $H^{2}\left(S^{2} \times R P^{2}, \mathbb{Z}_{2}\right), l \cup l=l^{2}=0$ in $H^{4}\left(S^{2} \times R P^{2}, \mathbb{Z}_{2}\right)$, but there is $\delta$ in $H^{2}\left(S^{2} \times S^{2} / \simeq, \mathbb{Z}_{2}\right)$ such that $\delta^{2}$ is not equal to 0 . First, by the Künneth formula,

$$
\begin{aligned}
& H^{2}\left(R P^{2} \times S^{2}, \mathbb{Z}_{2}\right) \\
& \quad=H^{2}\left(R P^{2}, \mathbb{Z}_{2}\right) \otimes H^{2}\left(S^{2}, \mathbb{Z}\right) \oplus H^{2}\left(R P^{2}, \mathbb{Z}_{2}\right) \otimes H^{2}\left(S^{2}, \mathbb{Z}\right) \\
& \quad=\left(\mathbb{Z}_{2} \otimes \mathbb{Z}\right) \oplus\left(\mathbb{Z}_{2} \otimes \mathbb{Z}\right)=\mathbb{Z}_{2} \oplus \mathbb{Z}_{2} .
\end{aligned}
$$

If $a$ is represented by $S^{2} \times\left\{y_{0}\right\}$ where $y_{0}$ is a base point of $R P^{2}$, and $b$ is represented by $\left\{x_{0}\right\} \times R P^{2}$, where $x_{0}$ is the north pole of $S^{2}$, then $a \cup$ $a=a^{2}=b \cup b=b^{2}=0$, since we can deform $\left\{x_{0}\right\} \times R P^{2}$, and $S^{2} \times\left\{y_{0}\right\}$ slightly so that there is no self-intersection respectively. Since any element $\tau$ in $H^{2}\left(R P^{2} \times S^{2}, \mathbb{Z}_{2}\right)$ can be expressed as $\tau=m a+n b$, where $0 \leq m, n \leq 1$, $\tau=(m a+n b)^{2}=m^{2} a^{2}+2 m n a b+n^{2} b^{2}=2 m n a b=0$, (the coefficient is $\mathbb{Z}_{2}$ ).

On the other hand,

$$
\begin{gathered}
R P^{2} \stackrel{\text { incl }}{\hookrightarrow} S^{2} \times S^{2} / \simeq \stackrel{\text { proj }}{\longrightarrow} R P^{2}, \\
{[\nu] \mapsto[(x, \nu)] \mapsto[\nu],}
\end{gathered}
$$

where incl is the inclusion and proj is the natural projection. Then proj $\circ$ incl $=$ Id,

$$
H_{2}\left(R P^{2}, \mathbb{Z}_{2}\right) \stackrel{\text { (incl)* }}{\longrightarrow} H_{2}\left(S^{2} \times S^{2} / \simeq, \mathbb{Z}_{2}\right) \stackrel{(\text { (proj) })^{*}}{\longrightarrow} H_{2}\left(R P^{2}, \mathbb{Z}_{2}\right)
$$

and $(\text { proj })_{*} \circ(\text { incl })_{*}=$ Id . For $(\text { incl })_{*}(\alpha)$ in $H_{2}\left(S^{2} \times S^{2} / \simeq, \mathbb{Z}_{2}\right)$, by Poincaré duality, there is the corresponding class $\kappa$ in $H^{2}\left(S^{2} \times S^{2} / \simeq, \mathbb{Z}_{2}\right)$, where $\alpha$ in $H_{2}\left(R P^{2}, \mathbb{Z}_{2}\right)$ is the nontrivial element. We want to prove $\kappa^{2}$ is not zero. Since $\kappa^{2}$ is the geometric self-intersection number of $(\mathrm{incl})_{*}(\alpha)(\bmod 2)$, we have only to prove that the self-intersection number of the base space $R P^{2}$ of $S^{2} \times$ $S^{2} / \simeq$ is nonzero $(\bmod 2)$. We want to deform the base space $R P^{2}$ to get the self-intersection number. So we have only to consider a tubular neighborhood of $R P^{2}$ which is diffeomorphic to $R^{2} \times S^{2} / \simeq$, where $\simeq$ means $\left.((x, y), \nu)\right)$ is identified with $((-x,-y),-\nu))$. In other words, we have only to count the self-intersection numbers of $R P^{2}$ in $R^{2} \times S^{2} / \simeq$. The self-intersection number can be computed as follows: Note that $R^{2} \times S^{2} / \simeq=R \times S^{2} / \simeq \oplus R \times S^{2} / \simeq$ where $\oplus$ is the Whitney sum of the vector bundles and $\simeq$ in $R \times S^{2}$ means $(x, \nu)$ is identified with $(-x,-\nu)$. Since $R \times S^{2} / \simeq$ is the tautological bundle over $R P^{2}$ which is nontrivial (cf. [MS, p. 38]), if we let $R \times S^{2} / \simeq=\gamma_{2}^{1}$ the mod 2 self-intersection of the base space $R P^{2}$ is the second Stiefel-Whitney class of $R^{2} \times S^{2} / \simeq$. The total class is

$$
\begin{aligned}
\omega\left(\gamma_{2}^{1} \oplus \gamma_{2}^{1}\right) & =\omega\left(\gamma_{2}^{1}\right) \omega\left(\gamma_{2}^{1}\right)=\left(1+\omega_{1}\left(\gamma_{2}^{1}\right)\right)\left(1+\omega_{1}\left(\gamma_{2}^{1}\right)\right) \\
& =1+2 \omega_{1}\left(\gamma_{2}^{1}\right)+\omega_{1}^{2}\left(\gamma_{2}^{1}\right) .
\end{aligned}
$$


So

$$
\omega_{2}\left(R^{2} \times S^{2} / \simeq\right)=\omega_{2}\left(\gamma_{2}^{1} \oplus \gamma_{2}^{1}\right)=\omega_{1}^{2}\left(\gamma_{2}^{1}\right) .
$$

Since $\omega_{1}^{2}\left(\gamma_{2}^{1}\right)$ is the nonzero element in $H^{2}\left(R P^{2}, \mathbb{Z}_{2}\right)$ (cf. [MS, p. 47]), $\omega_{2}\left(\gamma_{2}^{1} \oplus \gamma_{2}^{1}\right)=1$. This implies $\kappa^{2}$ is not zero $(\bmod 2)$.

(3.7) Theorem. $S^{2} \times S^{2} / \simeq$ is not homotopy equivalent to $M_{2} \quad\left(=D^{2} \times S^{2} / \simeq\right.$ $\left.\cup_{g_{2}} D^{2} \times S^{2} / \simeq\right)$.

To prove (3.7), we need a homotopy invariant which was discovered recently. In Homotopy invariants of nonorientable 4-manifolds (see [KKR]), the invariant was defined as follows: Choose an element $x$ in $\Pi_{2}(M)$, and represent it by a transversally immersed 2-sphere $S_{x}$. Then fixing an orientation of $\widetilde{M}$ (the orientable double covering of $M$ ), define a function $q$ with values in $\mathbb{Z}_{4}$ by

$$
\mathscr{X}\left(\nu\left(S_{x}\right)\right)+2 \# \text { self } S_{x}(\bmod 4) .
$$

Here $\mathscr{X}\left(\nu\left(S_{x}\right)\right)$ is Euler number of the pull-back bundle $f^{*}\left(\nu\left(S_{x}\right)\right)$ with respect to the orientation induced from $\widetilde{M}$. Here $f$ is a transversally immersed map from $S^{2}$ to $M, \nu\left(S_{x}\right)$ is a normal bundle over $S_{x}$ in $M$ and \# self $S_{x}$ means the self-intersection cardinal number $S_{x}$ in $M$.

Now we are ready to state the main theorem in that paper without proof.

Theorem. Suppose that $f$ is a homotopy equivalence from $M$ to $N$. Then $q_{M}(x)=q_{N}\left(f_{*}(x)\right)$, for all $x$ in $\Pi_{2}(M)$, with respect to relevant orientations of the orientable double covers $M$ and $N$.

Proof of (3.7). Suppose there exists a homotopy equivalence $f$ from $S^{2} \times S^{2} / \simeq$ to $M_{2}$. Then we have

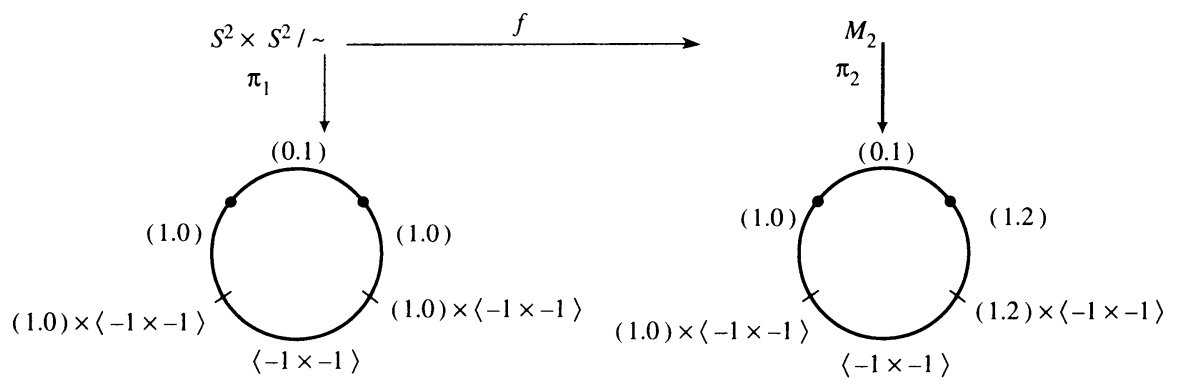

where $\pi_{1}, \pi_{2}$ are the natural projections.

Consider the orientable double covering $S^{2} \times S^{2}$ with the induced $T^{2}$-actions. Then we have orbit spaces as shown in Figure 4 corresponding to those shown in Figure 5 respectively. Choose an element $x$ in $\Pi_{2}\left(S^{2} \times S^{2} / \simeq\right.$ ) (the second homotopy group of $S^{2} \times S^{2} / \simeq$ ) which corresponds to the preimage of the arc $A$ (i.e. $\left.\pi_{1}^{-1}(A)\right)$ in $S^{2} \times S^{2} / \simeq$. Then $q_{M}(x)=0+0=0(\bmod 4)$.

Next we want to show that $f_{*}(x)$ in $\Pi_{2}\left(M_{2}\right)$ is represented by $\pi_{2}^{-1}(B)$ of the $\operatorname{arc} B$ in Figure 6 let

$$
U_{1}=\left\{(x, y): x^{2}+y^{2} \leq 1, x<1 / 2\right\}
$$

and

$$
U_{2}=\left\{(x, y): x^{2}+y^{2} \leq 1, x>-1 / 2\right\}
$$



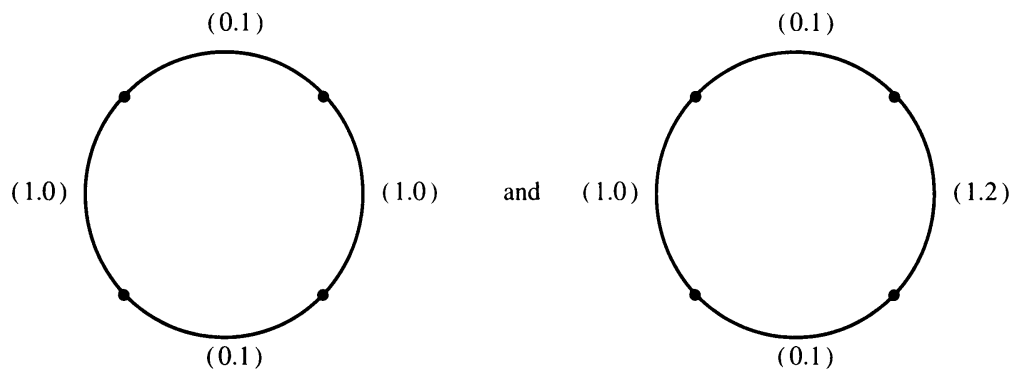

FIGURE 4
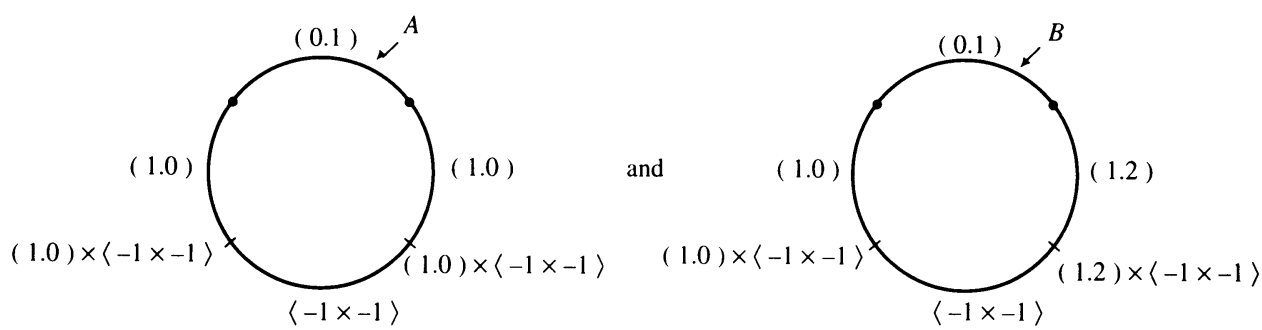

Figure 5

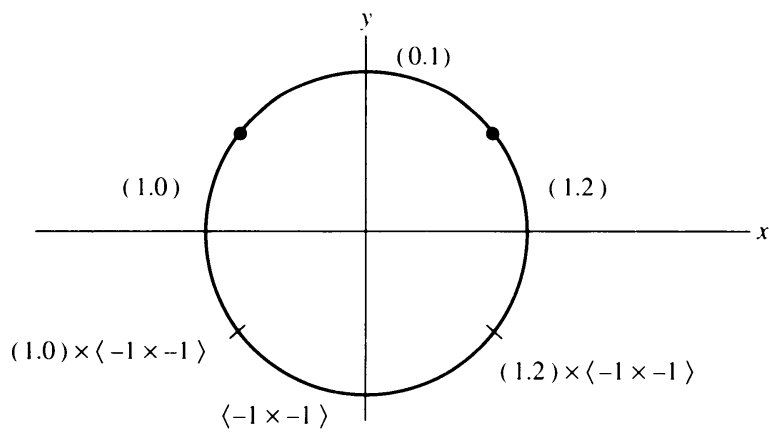

Figure 6

Note that $\pi_{2}^{-1}\left(U_{1}\right)=\pi_{2}^{-1}\left(U_{2}\right)=\stackrel{\circ}{D^{2}} \times S^{2} / \simeq$ where $\stackrel{\circ}{D}^{2}=$ the interior of $D^{2}$ and $\pi_{2}^{-1}\left(U_{1} \cap U_{2}\right)$ is homotopy equivalent to $S^{1} \times S^{2} / \simeq$ from (3.4). By the Mayer-Vietoris sequence, we have

$$
\begin{gathered}
H_{2}\left(\pi_{2}^{-1}\left(U_{1}\right)\right) \oplus H_{2}\left(\pi_{2}^{-1}\left(U_{2}\right)\right) \rightarrow H_{2}\left(M_{2}\right) \stackrel{\delta *}{\longrightarrow} H_{1}\left(S^{1} \times S^{2} / \simeq\right) \\
\rightarrow H_{1}\left(\pi_{2}^{-1}\left(U_{1}\right)\right) \oplus H_{1}\left(\pi_{2}^{-1}\left(U_{2}\right)\right) \rightarrow H_{1}\left(M_{2}\right) \rightarrow 0
\end{gathered}
$$

where $\delta_{*}$ is the boundary homomorphism. Since $\pi_{2}^{-1}\left(U_{1}\right)$ and $\pi_{2}^{-1}\left(U_{2}\right)$ have the same homotopy type as $R P^{2}$ and the fundamental group of $S^{1} \times S^{2} / \simeq$ is 
$\mathbb{Z}$, we obtain the exact sequence

$$
0 \rightarrow H_{2}\left(M_{2}\right) \rightarrow \mathbb{Z} \rightarrow \mathbb{Z}_{2} \oplus \mathbb{Z}_{2} \rightarrow H_{1}\left(M_{2}\right) \rightarrow 0 .
$$

Since $H_{1}\left(M_{2}\right)=\mathbb{Z}, H_{2}\left(M_{2}\right)$ is a subgroup of $\mathbb{Z}$ and nonzero, so $H_{2}\left(M_{2}\right)=\mathbb{Z}$. The generator of $H_{1}\left(S^{1} \times S^{2} / \simeq\right)$ is represented by $\pi_{2}^{-1}(P)$. By the definition of $\delta_{*}, \pi_{2}^{-1}(B)$ represents the generator of $H_{2}\left(M_{2}\right)$. By the Hurewicz homomorphisms and their naturality, i.e.

$$
\begin{array}{cc}
\Pi_{2}\left(S^{2} \times S^{2} / \simeq\right) & \longrightarrow H_{2}\left(S^{2} \times S^{2} / \simeq\right)=\mathbb{Z} \\
f_{*} \downarrow \cong & f_{*} \downarrow \cong \\
\Pi_{2}\left(M_{2}\right) & \longrightarrow H_{2}\left(M_{2}\right)=\mathbb{Z}
\end{array}
$$

$f_{*}(x)$ in $\Pi_{2}\left(M_{2}\right)$ is represented by $\pi_{2}^{-1}(B)$, and $\pi_{2}^{-1}(B)$ corresponds to $\tilde{\pi}_{2}^{-1}(\widetilde{B})$ in $S^{2} \times S^{2}$, where $\widetilde{B}$ is an arc as shown below:

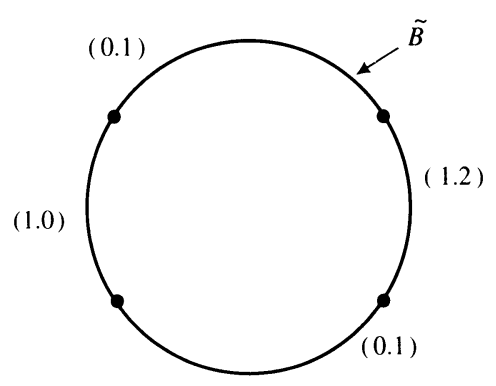

According to [OR, I], the Euler number of the normal bundle of $\tilde{\pi}_{2}^{-1}(\widetilde{B})$ is the determinant $\left|\begin{array}{ll}1 & 0 \\ 1 & 2\end{array}\right|$ with a sign. We have $\mathscr{X}\left(\nu\left(S_{f_{*}(x)}\right)\right)=+2$ or -2 . Since there is no self-intersection point in $M$ as we can see

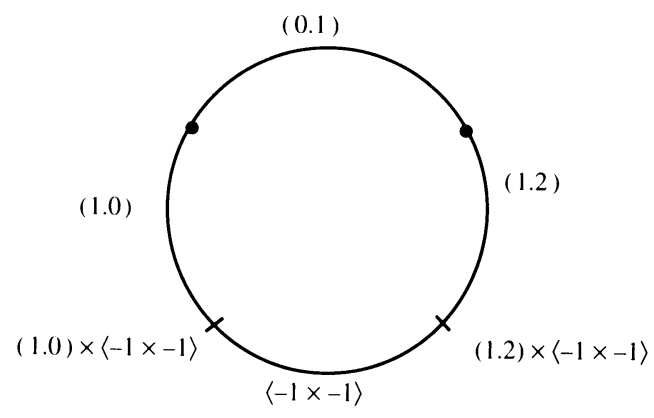

we have $q_{M_{2}}\left(f_{*}(x)\right) \equiv 2(\bmod 4)$. We have a contradiction. We can conclude that those are not homotopy equivalent. 


\section{STABLE DIFFEOMORPHISM THEOREM}

In this section, we are going to prove the stable diffeomorphism theorem (4.4). If $M$ is closed, nonorientable and admits a toral action with fixed points and no $\mathbb{Z}_{2} \times \mathbb{Z}_{2}$ stabilizer, then $M$, after blowing up with $\mathbb{C P}^{2}, s$, is diffeomorphic to a connected sum of 8 basic $T^{2}$-manifolds and 2 families of $T^{2}$-manifolds. To prove the theorem, we need the following lemma:

(4.1) Lemma. Suppose $M^{*}$ is given as below (only one $\mathbb{Z}_{2}$ component):

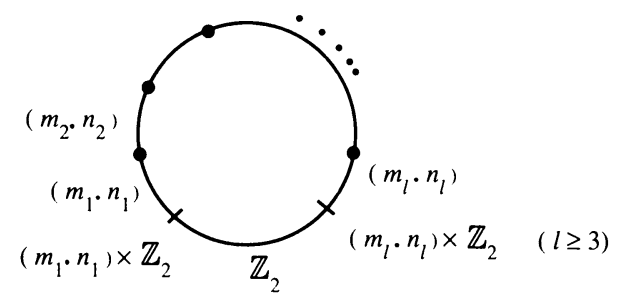

Then $M \# n \mathbb{C P}^{2}$ can be expressed as a connected sum of $S^{2} \times S^{2}, \mathbb{C P} P^{2}, R P^{2} \times S^{2}$, where $n$ is some integer.

Proof. By reparametrizing $T^{2}$, we may assume that $M^{*}$ is one of the following types:
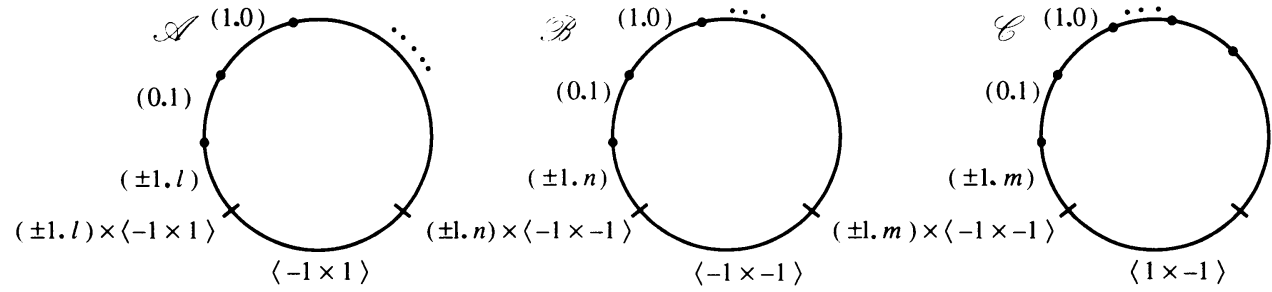

Recall that $n$ and $m$ must be even and odd in $\mathscr{B}$ and $\mathscr{C}$ respectively.

As in $\S 2$, we break the orbit space of each type into two pieces and investigate them as follows:

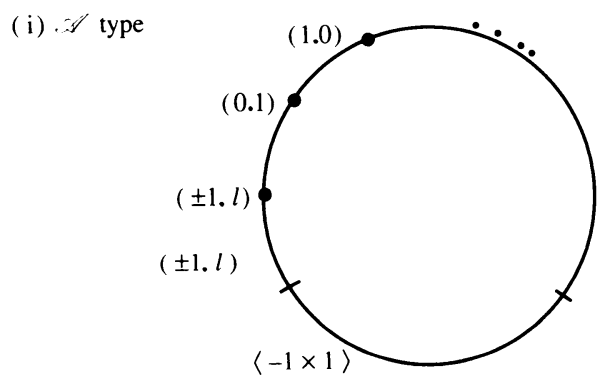

where $A_{1}$ and $A_{2}$ are 4-manifolds. 
Then, by applying the same methods as in (3.2), $M$ is diffeomorphic to a manifold $\tilde{A}_{i}(i=1$ or 2$)$ with orbit spaces
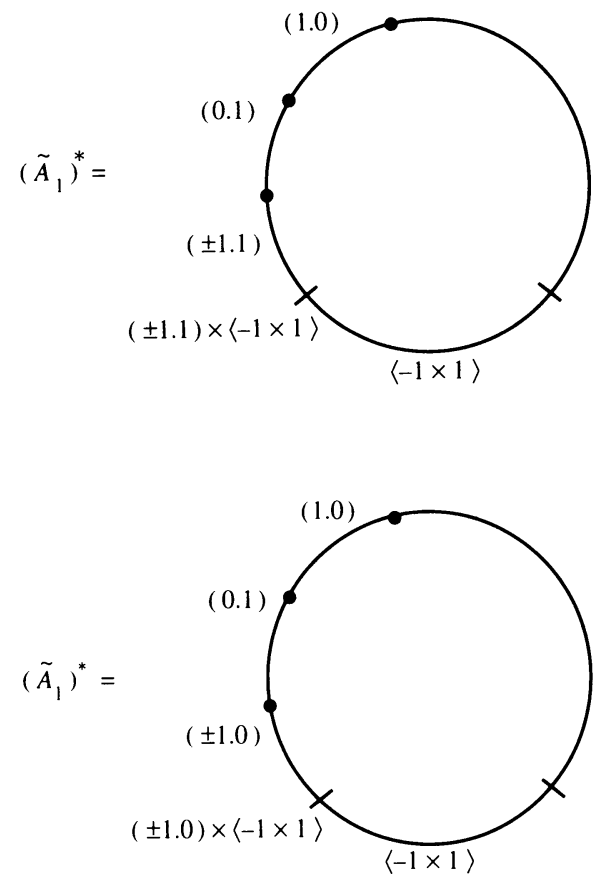

according as $l$ is odd or even respectively. We can reduce the number of fixed points i.e. we can express $\widetilde{A_{i}}$ as a connected sum of a simply-connected manifold and some manifold $K$ whose number of fixed points is less than that of $\widetilde{A_{i}}$ as shown below
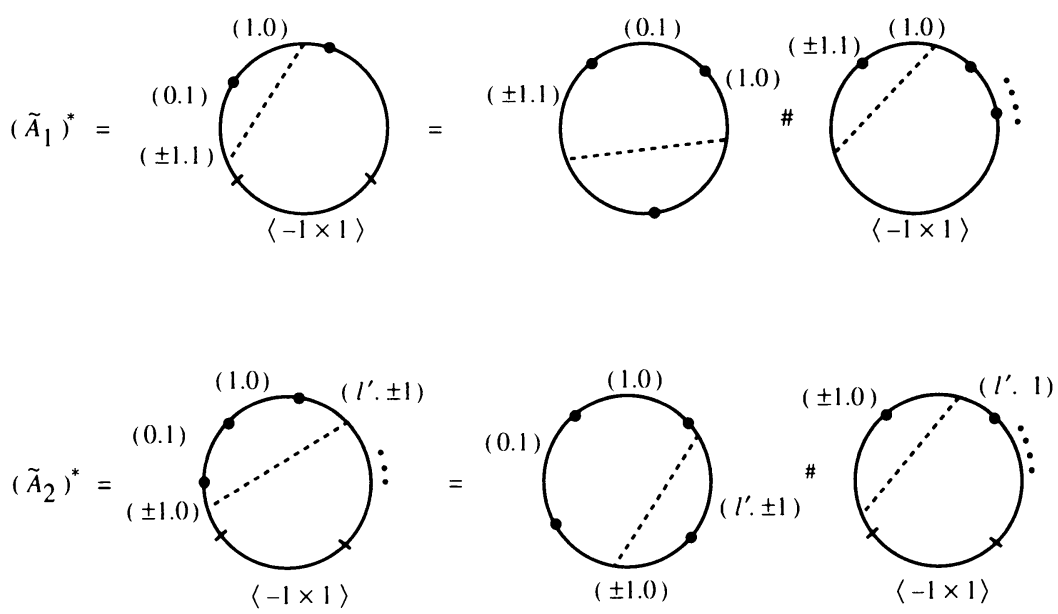
If we get type $\mathscr{A}$, then we apply the above method again. Suppose $K_{i}$ is of type $\mathscr{B}$ or $\mathscr{C}$. By adding

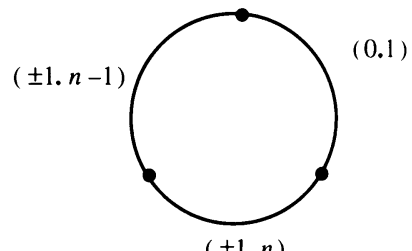

to

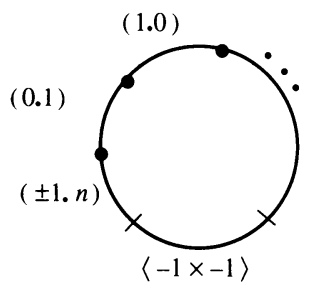

and
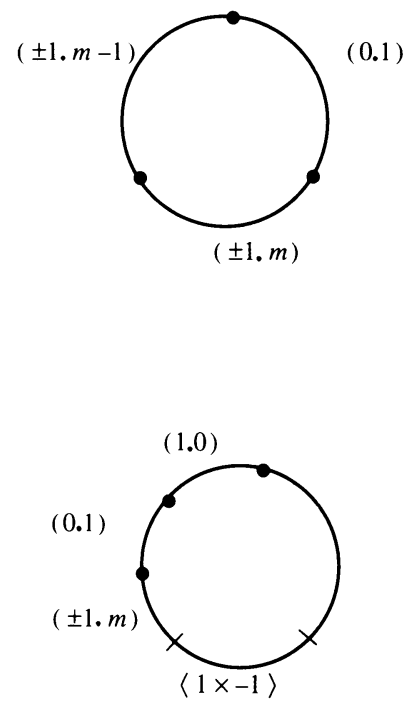

respectively, we have
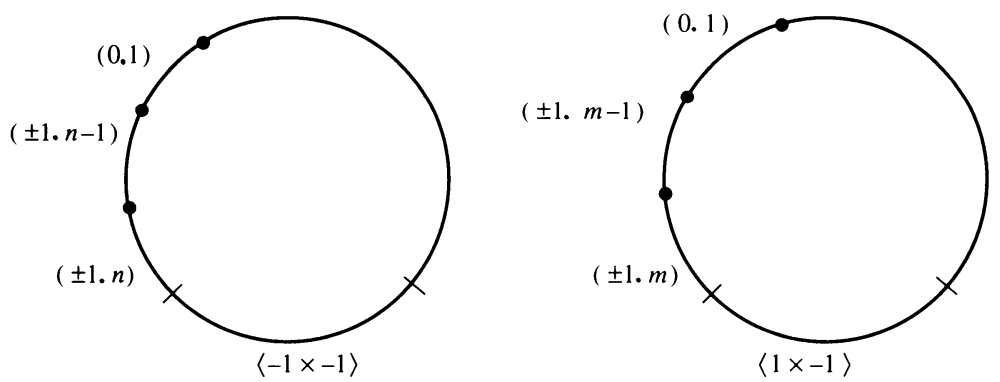

Then $\langle-1 \times-1\rangle$ is contained in $(1 . n-1)$, and $\langle 1 \times-1\rangle$ is contained in $(1 . m-1)$. By an automorphism of $T^{2}$, we get type $\mathscr{A}$ and apply the above argument until we get less than three fixed points. By (3.3) and (3.5), we obtain the desired result.

(4.2) Lemma. Suppose $M^{*}$ is given as follows:

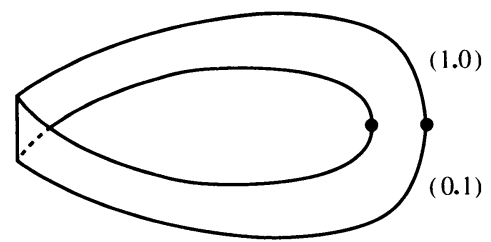


Then $M$ is diffeomorphic to $S^{2} \times S^{2} \#\left(S^{1} \times S^{3} / \simeq\right)$ where $\simeq$ means $(\alpha,(x, y$, $z, w))$ is identified with $(-\alpha,(x, y, z,-w))$.

Proof. $M^{*}$ can be obtained as follows: Consider $N^{*}$ as shown below:

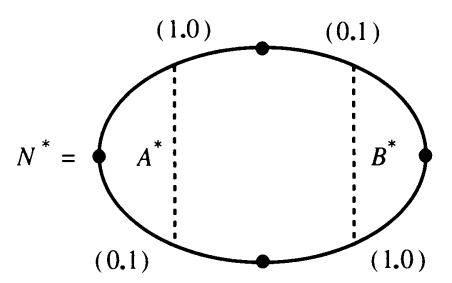

Note that $N=S^{2} \times S^{2}$. Delete $A^{*}$ and $B^{*}$ whose preimage are 4-dimensional disks and attach $\partial A^{*}$ and $\partial B^{*}$ by using an orientation preserving diffeomorphism. So, in the total spaces, two boundaries of $C$ are $S^{3}$. If we identify $S^{3}$ with the other $S^{3}$ by using an orientation preserving map $f$ from $\cdot S^{3}$ to $S^{3}$, we get $M$. Thus $M=S^{2} \times S^{2} \#\left(S^{3} \times S^{1} / \simeq\right)$.

(4.3) Lemma. Suppose we have the following orbit space:

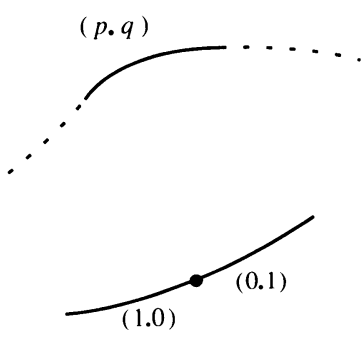

where neither $p$ nor $q$ are \pm 1 . By adding some number of $\mathbb{C P}^{2}$ 's or $\overline{\mathbb{C P}}^{2}$ 's to the total space, we can make the resulting orbit space as follows:

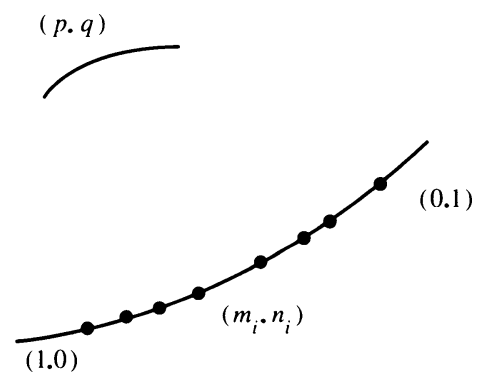


such that, for some $m_{i} n_{i}$,

$$
\left|\begin{array}{cc}
m_{i} & n_{i} \\
p & q
\end{array}\right|=1 \text { or }-1
$$

Proof. This can be proved easily by using the Euclidean algorithm.

Remark. Suppose $M^{*}$ is as shown below:

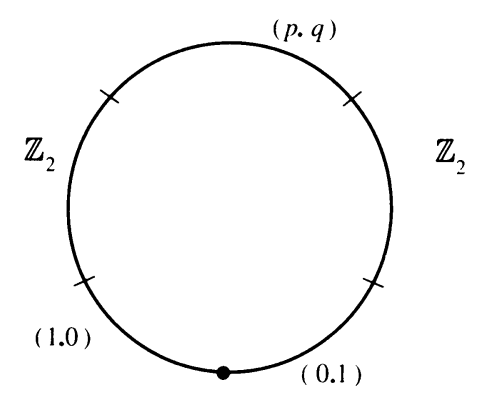

Then, by using (4.3), we get

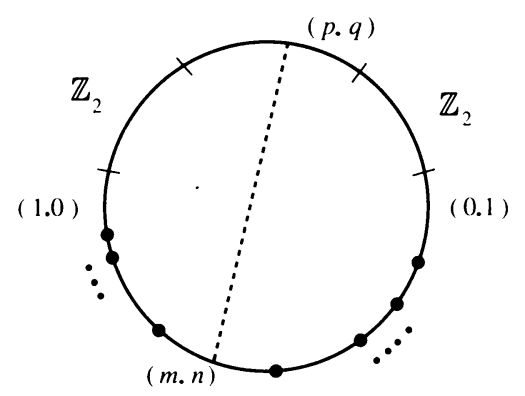

such that $\left|\begin{array}{ll}p & q \\ m & n\end{array}\right|= \pm 1$. We decompose this into two orbit spaces
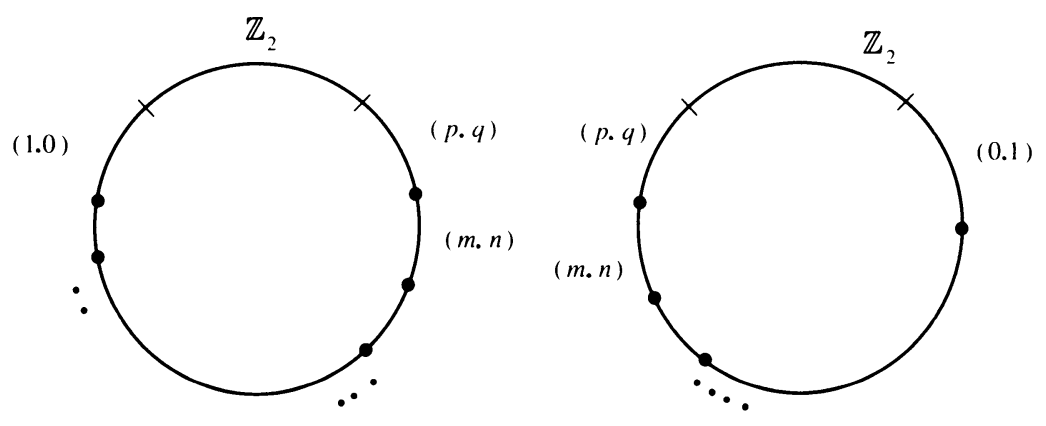

so that each of them has only one $\mathbb{Z}_{2}$ component.

We are ready to prove the stable diffeomorphism theorem.

(4.4) Theorem. Suppose $M^{*}$ has no $\mathbb{Z}_{2} \times \mathbb{Z}_{2}$ stabilizer on each of the boundary and has a fixed point. Then $M \# n \mathbb{C P}^{2}$ can be expressed as a connected sum of $S^{2} \times S^{2}, \mathbb{C P}^{2}, R P^{2} \times S^{2}, S^{1} \times S^{3} / \simeq, L_{n}, L_{n}^{\prime}, S^{1} \times S^{3}, K$, where $n$ is some positive integer, $L_{n}, L_{n}^{\prime}$ are 4-manifolds whose orbit spaces are (respectively) 

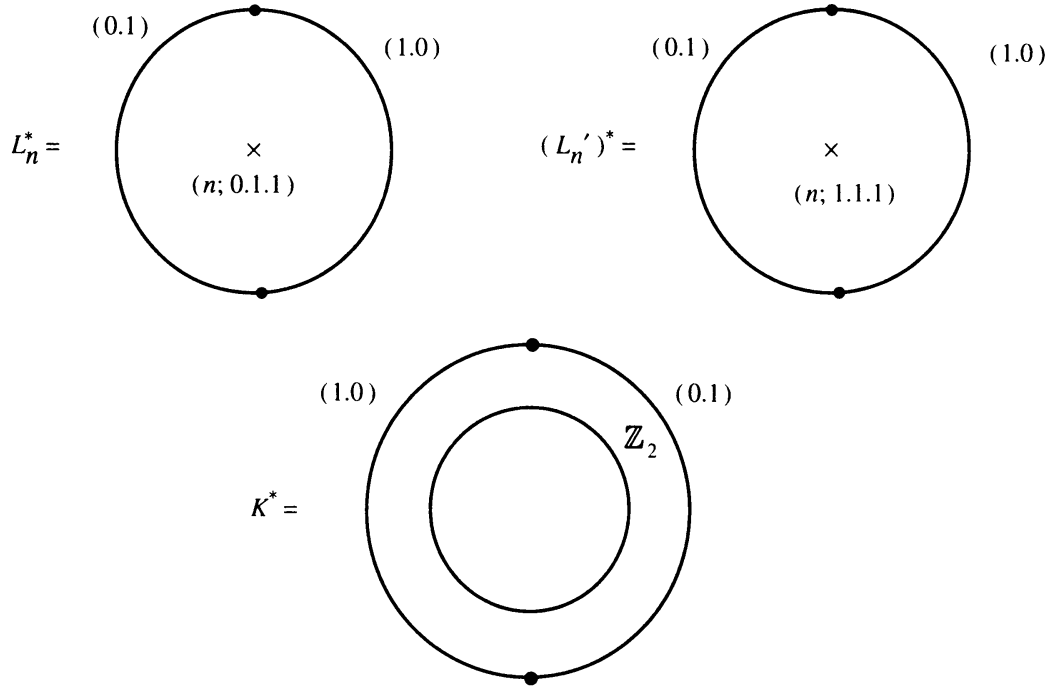

(see $[\mathrm{P}]$ for more details).

Proof. Since $M^{*}$ has a fixed point, $M^{*}$ is a 2-dimensional manifold with nonempty boundary. The proof follows from Theorem VI.1 of [P] and (2.7). $\quad$

\section{Remark.}
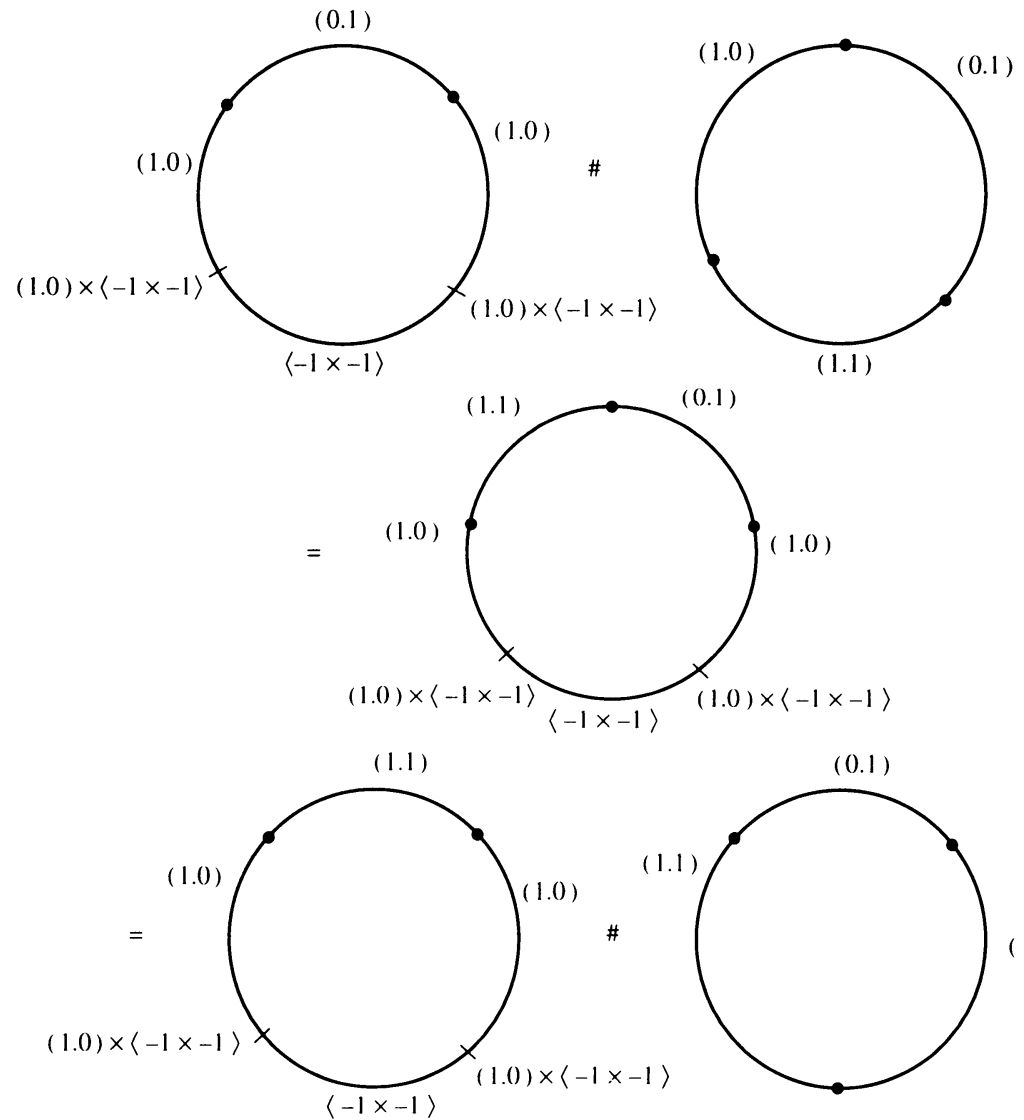
By reparametrizing $T^{2}$, we get

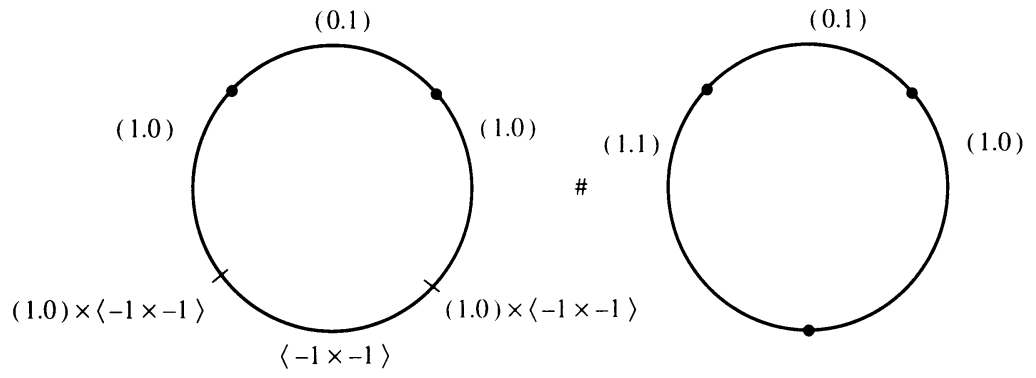

Thus $S^{2} \times S^{2} / \simeq \# \mathbb{C P}^{2}=R P^{2} \times S^{2} \# \mathbb{C P}^{2}$, even if $S^{2} \times S^{2} / \simeq$ is not homotopy equivalent to $R P^{2} \times S^{2}$. In the same way, we can obtain $M_{2} \# \mathbb{C P}^{2}=R P^{2} \times$ $S^{2} \# \mathbb{C P}^{2}$.

\section{REFERENCES}

[B] G. Bredon, Introduction to compact transformation groups, Academic Press, New York, 1972.

[H] M. Hirsch, Differential topology, Springer-Verlag, Berlin and New York, 1976.

[KR] M. Ho Kim and F. Raymond, The diffeotopy group of the twisted 2-sphere bundle over the circle, Trans. Amer. Math. Soc. 322 (1990), 159-168.

[KKR] M. Ho Kim, S. Kojima, and F. Raymond, Homotopy invariants of nonorientable 4-manifolds, Trans. Amer. Math. Soc. 333 (1992), 71-82.

[MS] J. Milnor and J. Stasheff, Lectures on characteristic classes, Princeton Univ. Press, Princeton, N.J., 1974.

[M] J. Munkres, Topology. A first course, Prentice-Hall, Englewood Cliffs, N.J., 1975.

[OR, I] P. Orlik and F. Raymond, Actions of the torus on 4-manifolds. I, Trans. Amer. Math. Soc. 152 (1970), 531-559.

[OR, II] _ Actions of the torus on 4-manifolds. II, Topology 13 (1974), 89-112.

[P] P. Pao, The topological structure of 4-manifolds with effective toral actions. I, Trans. Amer. Math. Soc. 227 (1977), 279-317.

Department of Mathematics, Sungkyunkwan University, Suwon, Kyungkido, Korea 440-746 